\title{
RADIO-X-RAY SYNERGY TO DISCOVER AND STUDY JETTED TIDAL DISRUPTION EVENTS
}

\author{
I. Donnarumma ${ }^{1}$ and E. M. Rossi ${ }^{2}$ \\ ${ }^{1}$ INAF-IAPS, Via Fosso del Cavaliere 100, I-00133, Rome, Italy; immacolata.donnarumma@iaps.inaf.it \\ ${ }^{2}$ Leiden Observatory, Leiden University, P.O. Box 9513, 2300 RA, Leiden, The Netherlands \\ Received 2014 July 11; accepted 2015 February 9; published 2015 April 13
}

\begin{abstract}
Observational consequences of tidal disruption of stars by supermassive black holes (SMBHs) can enable us to discover quiescent SMBHs, constrain their mass function, and study the formation and evolution of transient accretion disks and jet formation. A couple of jetted tidal disruption events (TDEs) have been recently claimed in hard X-rays, challenging jet models, which were previously applied to $\gamma$-ray bursts and active galactic nuclei. It is therefore of paramount importance to increase the current sample. In this paper, we find that the best strategy is not to use upcoming X-ray instruments alone, which will yield between several (eRosita) and a couple of hundred (Einstein Probe) events per year below redshift one. We rather claim that a more efficient TDE hunter will be the Square Kilometer Array (SKA) operating in survey mode at $1.4 \mathrm{GHz}$. It may detect up to several hundred events per year below $z \sim 2.5$ with a peak rate of a few tens per year at $z \approx 0.5$. Therefore, even if the jet production efficiency is not $100 \%$ as assumed here, the predicted rates should be large enough to allow for statistical studies. The characteristic TDE decay of $t^{-5 / 3}$, however, is not seen in radio, whose flux emission is quite featureless. Identification therefore requires localization and prompt repointing by higher energy instruments. If radio candidates would be repointed within a day by future X-ray observatories (e.g., Athena- and LOFT-like missions), it will be possible to detect up to $\approx 400 \mathrm{X}$-ray counterparts, almost up to redshift 2 . The shortcoming is that only for redshift below $\approx 0.4$ will the trigger times be less than 10 days from the explosion. In this regard the $\mathrm{X}$-ray surveys are better suited to probe the beginning of the flare, and are therefore complementary to SKA.
\end{abstract}

Key words: black hole physics - galaxies: nuclei - hydrodynamics

\section{INTRODUCTION}

Since the late 1970s it has been suggested that stars torn apart by the gravitational field of a supermassive black hole (SMBH) may be observed as flares from the Earth (Hills 1975; Frank \& Rees 1976; Rees 1988; Phinney 1989). These are called tidal disruption events (TDEs). These flares would be caused by sudden accretion of the star debris, which would feed the SMBH at an ever decreasing rate, $\dot{M} \propto t^{-5 / 3}$. This theoretical expectation is for a complete disruption of a star in parabolic orbit, after at least several days from the peak (e.g., Lodato et al. 2009; Guillochon \& Ramirez-Ruiz 2013; Hayasaki et al. 2013), and it is expected to be independent on the ratio of pericenter to tidal radius (Sari et al. 2010; Stone et al. 2013).

The detection and study of these flares can deliver important astrophysical information. On the one hand, they allow us to detect otherwise quiescent SMBHs and to estimate their masses. This would inform theory of galaxy-SMBH cosmological co-evolution. On the other hand, they constitute a unique opportunity to study the-highly theoretically uncertain - formation of an accretion disk and its continuous transition through different accretion states. As the accretion rate decreases, we can in principle observe a disk that transits from an initial super-Eddington phase, lasting several months, passing through a slim and later a thin disk regime, and ending its life, years later, in a radiative inefficient state. The superEddington phase-which occurs only for SMBH masses $\lesssim 10^{7} M_{\odot}$-is highly uncertain, but it may be associated with a copious radiative driven wind (Rossi \& Begelman 2009), which thermally emits $\sim 10^{41}-10^{43} \mathrm{erg} \mathrm{s}^{-1}$, mainly at optical frequencies (Strubbe \& Quataert 2009; Lodato \& Rossi 2011). The disk luminosity $\left(\sim 10^{45}-10^{46} \mathrm{erg} \mathrm{s}^{-1}\right)$, instead, peaks in
far-UV/soft X-rays (Lodato \& Rossi 2011). Of paramount theoretical importance would also be the possibility to investigate the formation and evolution of an associated jet powered by this sudden accretion. There is no specific theory for the jet emission from TDEs. Astronomers mainly assume a phenomenological description (e.g., Cannizzo et al. 2011; van Velzen et al. 2011) or borrow theory developed for blazars and/or $\gamma$-ray bursts (e.g., Metzger et al. 2012; Tchekhovskoy et al. 2013). In general, non-thermal emission in Xrays and radio is the jet signature.

A handful of candidate TDEs $(\sim 10)$ have been detected so far, particularly in the ROSAT all-sky survey (Komossa 2002; Donley et al. 2002), in the GALEX Deep Imaging Survey (Gezari et al. 2009, 2012; Campana 2011), and in SDSS (van Velzen et al. 2011a). These "soft" events are believed to be associated with the disk and wind thermal emission. The presence of a bright optical flare in the initial super-Eddington months makes optical surveys a useful tool for discovery. Significant advances in optical transient surveys are expected to be achieved by the Panoramic Survey Telescope and Response System (Pan-STARRS) and the Large Synoptic Survey Telescope (LSST). Two candidates have been claimed in Pan-STARRS data (Gezari et al. 2012; Chornock et al. 2014), three in PTF data (Arcavi et al. 2014), and two in ASAS-SN (Holoien et al. 2014; Jose et al. 2014; Miller et al. 2014), but the total number expected seems to be much higher. For example in the $3 \pi$ Survey, claims in the literature range from 200 to $\sim 1557$, while in the medium deep survey there is more consensus that $\sim 15-20$ should be found (Strubbe \& Quataert 2009; van Velzen et al. 2011a). Thousands of candidates could be, instead, detected by LSST, with its six-band (0.3-1.1 micron) wide-field deep astronomical survey of over 20,000 square degrees of the southern sky, using an $8.4 \mathrm{~m}$ ground- 


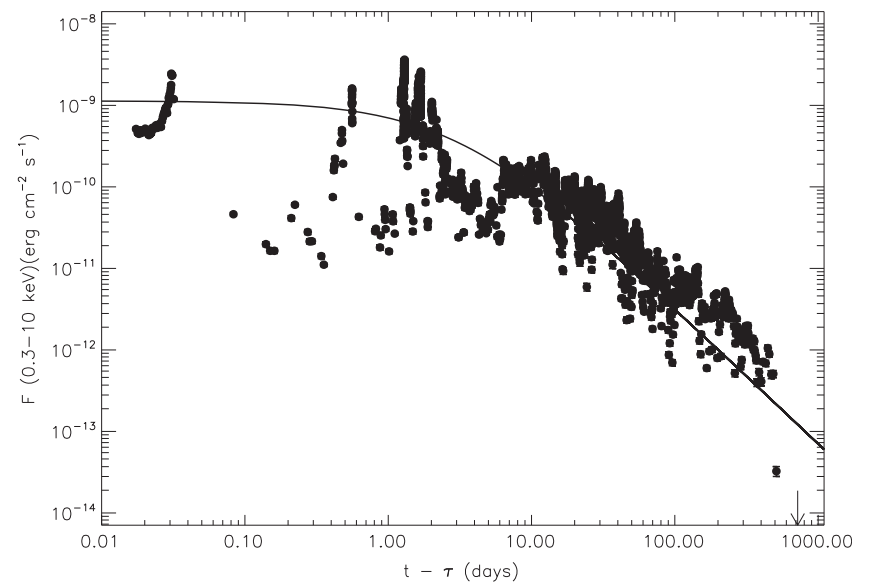

Figure 1. X-ray $(0.3-10 \mathrm{keV})$ light curve of J1644 as a function of time from the X-ray trigger: data (absorbed flux, circle marks taken from publicly available XRT light curves) vs. our modeling (solid line). After a few days, the temporal decay approaches $t^{-5 / 3}$.

based telescope (Strubbe \& Quataert 2009; van Velzen et al. 2011a). However, these estimates are probably upper limits because galactic nuclei can heavily absorb optical light.

More recently, two candidate TDEs were triggered in the hard X-ray band by the BAT instrument on board Swift (Bloom et al. 2011; Burrows et al. 2011; Cenko et al. 2012). A multifrequency follow-up from radio to $\gamma$-rays revealed a new class of TDEs, where we are likely observing the non-thermal emission from a relativistic jet. The jet emission is responsible for the hard X-ray spectrum (with power-law slope $\beta \sim 1.7$ ) and the increasing radio activity (Levan et al. 2011), which was detected a few days after the trigger.

Given the lack of statistics and of a solid theoretical framework for the non-thermal emission, we will take the best studied of these two events, Swift J1644+57 (Sw J1644 in short), as a prototype for the study presented in this paper, where we investigate the detection capability of both SKA and future X-ray observatories.

Sw J1644 was hosted by a star-forming galaxy at $z=0.354$ and in positional coincidence with its center (Zauderer et al. 2011). Its X-ray peak luminosity $\sim 3 \times 10^{48} \mathrm{erg} \mathrm{s}^{-1}$ was reached after a couple of days from the trigger, and it persisted at the level of $>10^{45} \mathrm{erg} \mathrm{s}^{-1}$ for about one year. During its decay, the X-ray emission was approximately described by a $t^{-5 / 3}$ temporal law, the same as that expected for the fallback of stellar debris (see Figure 1). After $\sim 500$ days from the trigger, the X-ray flux declined by two orders of magnitude and it has been associated with a shut-off of the relativistic jet (Zauderer et al. 2013). The modeling of the X-ray luminosity suggests that $\mathrm{Sw} \mathrm{J} 1644$ is associated with a light $\mathrm{SMBH} \lesssim 10^{7} M_{\odot}$ (e.g., Burrows et al. 2011; Cannizzo et al. 2011).

Variability at optical wavelengths within the host was not detected, while transient emission was seen in infrared, becoming stronger at longer wavelengths, especially at millimeter and radio wavelengths. Radio (1.4. and $4.8 \mathrm{GHz}$ ) observations from the Westerbork Synthesis Radio Telescope showed a bright source. EVLA observations of the radio transient coincident with the host galaxy were reported, providing an estimate of the bulk Lorentz factor of $\Gamma \sim 2$ of the outflow (Zauderer et al. 2011). The radio light curve displays a rebrightening starting one month after the trigger (Berger et al. 2012; Zauderer et al. 2013). The emission peaks around several months, followed by a decline. Radio observations stop at 600 days after the trigger (Zauderer et al. 2013). The radio behavior is not compatible with the blast wave model borrowed from $\gamma$-ray bursts by Metzger et al. (2012), and indicate a more complicated jet structure, perhaps as in the magnetically arrested model proposed by Tchekhovskoy et al. (2013). Snapshot rates of jetted TDEs in the radio band have been computed for the first time by van Velzen et al. (2011). In contrast to their work, we adopt here a different modeling for the radio light curve and a more detailed one for the black hole mass function, which includes the redshift dependence. We also account for a stellar mass function. We broaden up our investigation to include X-ray detection and follow-ups.

Finally, a $200 \mathrm{~s}$ quasi-periodic oscillation (QPO) was detected by both Suzaku and XMM, 10 and 19 days after the Swift/BAT trigger, respectively (Reis et al. 2012). QPOs are regularly detected in stellar mass BHs, but there is no firm physical interpretation of these phenomena. However, most models strongly link the origin of high-frequency QPOs with orbits or resonances in the inner accretion disk close to the $\mathrm{BH}$. This may cause variable energy injection into the jet, which consequently results in variability in the X-ray emission. This interpretation led Reis et al. (2012) to estimate a BH mass between $5 \times 10^{5} M_{\odot}$ and $5 \times 10^{6} M_{\odot}$ (Reis et al. 2012).

In this paper, we predict the detection rate of jetted TDEs considering current and future radio surveys (NVSS + FIRST, VLT Stripe 82, ASKAP, VLASS and SKA) and X-ray instruments (Swift, eRosita, Einstein Probe, Athena, and $L O F T$ ). In addition, we discuss the ability of these instruments to constrain important physical parameters.

The paper is organized as follows. In Section 2, we take Swift J1644 as a prototype and we describe our phenomenological model for X-ray and radio emissions. In Section 3, we discuss the black hole distribution functions used in this paper. In Section 4, we present our Monte Carlo calculations. Our rates for current and future surveys are presented in Section 5. A summary and the implications of our results can be found in Section 6. Finally, we draw our conclusions in Section 7.

Throughout this paper we use the following cosmological parameters: $\Omega_{\mathrm{M}}=0.25, \quad \Omega_{\lambda}=0.75$, and $H_{0}=$ $70 \mathrm{~km} \mathrm{~s}^{-1} \mathrm{Mpc}^{-1}$.

\section{MODELING THE LIGHT CURVE}

A tidal disruption event of a star by an SMBH causes a transient accretion disk to form, whose accretion rate is set by the rate at which the stellar debris falls back to the black hole under its gravitational pull. How matter circularizes to form a disk and whether this process is accompanied by outflows and their characteristics are subject to intense investigations, as mentioned above. From phenomenology and theory, we know that in the presence of an accretion disk and some ordered magnetic field, matter and energy outflows in form of (relativistic) jets are produced. In the absence of fully consistent simulations of jet production by a tidal disruption event, we use below a simplified description for the jet energy content as a function of time. This is partially supported by analytical and numerical calculations (see references above) and partly by the observed features of the X-ray emission of Sw J1644. In particular, its temporal decay $\left(\sim t^{-5 / 3}\right)$ suggests that at least in this optically thin regime, the X-ray luminosity scales as the accretion rate. As a consequence, it supports a scenario in which the star was completely tidally disrupted, since partial 
disruption would lead to a shallower decay of the fallback rate (Guillochon \& Ramirez-Ruiz 2013). Moreover, a partial disruption is difficult to reconcile with a long-lasting superEddington accretion phase, which may be needed to power the jet for its total duration of $\sim 500$ days. Finally, the modeling of the X-ray luminosity suggests that $\mathrm{Sw}$ J1644 is the consequence of a disruption of a roughly one solar mass star by a light $\mathrm{SMBH} \lesssim 10^{7} M_{\odot}$ (e.g., Burrows et al. 2011; Cannizzo et al. 2011).

\subsection{Jet Kinetic Power}

We work in the framework of two identical jets, with $\theta_{\mathrm{j}}<1 / \Gamma$. The total energy injected in the two jets is $L_{\mathrm{j}}=\epsilon_{\mathrm{j}} \dot{M}_{\mathrm{fb}} c^{2}$, where $\epsilon_{\mathrm{j}}$ is the jet production efficiency, which we assume constant in time, and the gas fall back to form a disk occurs at a rate $\dot{M}_{\mathrm{fb}}$. For a complete disruption of a star in parabolic orbit the fallback rate can be approximated by

$$
\dot{M}_{\mathrm{fb}}(\bar{\tau})=\dot{M}_{\mathrm{p}}\left(\frac{t_{\mathrm{min}}+\bar{\tau}}{t_{\min }}\right)^{-5 / 3},
$$

(Rees 1988; Phinney 1989). The lag time " $\bar{\tau}$ " is the time from the beginning of the debris accretion, which roughly happens after a time

$$
t_{\min } \approx 41 M_{6}^{1 / 2} m_{*, 1}^{1 / 2} \mathrm{day}
$$

from the star disruption, in the galaxy rest frame. More precisely, $t_{\min }$ is the minimum time it takes the most bound debris to come back to pericenter after the star has been torn apart. Here and in the following, $M_{6}$ is the $\mathrm{BH}$ mass in units of $10^{6} M_{\odot}$ and $m_{*, 1}$ the mass of the disrupted star in units of $1 M_{\odot}$. The peak of the accretion rate $^{3}$ is quite intuitively the mass of the star divided by the characteristic timescale, $\dot{M}_{\mathrm{p}} \approx(1 / 3) m_{*} / t_{\text {min }} \approx 1.9 \times 10^{26} M_{6}^{-1 / 2} m_{*, 1}^{1 / 2} \mathrm{~g} \mathrm{~s}^{-1}$. In our description, the jet is launched at the onset of accretion $(\bar{\tau}=0)$, as there are no strong theoretical reasons why it should be delayed. The temporal evolution of the jet energy is thus

$$
L_{\mathrm{j}}(\bar{\tau})=L_{\mathrm{j}, \mathrm{p}}\left(\frac{t_{\min }+\bar{\tau}}{t_{\min }}\right)^{-5 / 3}
$$

where

$$
L_{\mathrm{j}, \mathrm{p}}=\epsilon_{\mathrm{j}} \dot{M}_{\mathrm{p}} c^{2} \approx 1.7 \times 10^{45} \mathrm{erg} \mathrm{s}^{-1}\left(\frac{\epsilon_{\mathrm{j}}}{0.01}\right) M_{6}^{-1 / 2} m_{*, 1}^{1 / 2} .
$$

Note that the larger the black hole mass, the lower the peak luminosity because the characteristic timescale increases. Vice versa, the jet luminosity decreases with $m_{*}$.

\section{2. $X$-ray}

The unabsorbed 1-10 keV light curve of Sw J1644 is shown in Figure 1 (black circles). Activity was already detected by $\mathrm{BAT} \approx 3$ days before the BAT "official" trigger and the beginning of XRT observations (Burrows et al. 2011).

\footnotetext{
3 In the formula used in this paper, we assume the standard linear relation between mass and radius of the star. See Equation (6) in Lodato \& Rossi (2011).
}

Therefore there is an indication that the trigger (i.e., when the first photon was detected by XRT) happened approximately $\tau \approx 3$ days after the actual disk and jet formation. The observed time interval $\tau$ is related to the rest-frame analogous quantity by $\tau=\bar{\tau}(1+z)$ and in this case $\bar{\tau} \approx 2$ days. Accounting for this delay, the general behavior of the X-ray light curve as a function of time $\Delta t$ since the trigger $(\Delta t=0)$ can be reproduced by

$$
L_{\mathrm{x}, \text { iso }}(\Delta t) \approx 1.5 \times 10^{48} \operatorname{erg~s}^{-1}\left(\frac{\tau+\Delta t}{\tau}\right)^{-5 / 3},
$$

(Figure 1, solid line). Specifically, $L_{\mathrm{x}, \text { iso }}$ is an isotropic equivalent luminosity, computed from the X-ray flux. Note that here $\tau=3$ is a fixed time delay, unlike $\bar{\tau}$ in Equations (1) and (2). Superimposed to this baseline trend, there is a complex structure of flares and dips where the flux oscillates within two orders of magnitude in the first 10 days of observations. It is clear that Equation (4) does not capture this large variability, possibly associated with jet precession and nutation (Saxton et al. 2012; Stone \& Loeb 2012). But in absence of a compelling theory for these sudden X-ray variations, we prefer to reproduce the upper part of the envelope that contains the initial variability, since the BAT instrument was triggered by one of the peaks in the light curve. We will discuss later how this choice affects our X-ray TDE rate estimates.

The Swift/XRT (0.3-10 keV) spectrum of Sw J1644+57 is well described by an absorbed power law with a photon index $\beta \approx 1.7-1.8$ and $N_{\mathrm{H}} \approx 2 \times 10^{22} \mathrm{~cm}^{-2}$ (Burrows et al. 2011). The observed BAT spectrum at early times and its count rate later on (up to the beginning of June) are consistent with an extrapolation at higher energies of the XRT spectrum (Burrows et al. 2011). This suggests that we are observing the same component in both soft and hard X-ray bands. The average spectrum is consistently hard $(1.4 \lesssim \beta \lesssim 1.7)$ during the whole emission, although a spectral softening is observed during the short dips in the initial variable phase (Saxton et al. 2012). The radiation efficiency in the $1-10 \mathrm{keV}$ band (i.e., the fraction of the total luminosity emitted in that band) is $\epsilon_{\mathrm{x}} \approx 0.20$ (Burrows et al. 2011). With this last information, we can calculate the associated jet kinetic luminosity from the observed light curve, once we assume a jet opening angle $\theta_{\mathrm{j}}$ and a Doppler factor $\delta$,

$$
L_{\mathrm{j}}=L_{\mathrm{x}, \text { iso }}\left(1-\cos \theta_{\mathrm{j}}\right) /\left(\epsilon_{\mathrm{x}} \delta^{2}\right) \text {. }
$$

With the highest probability, our line of sight is at an angle $\sim \Gamma^{-1}$ (i.e., the inverse of the Lorentz factor $\Gamma$ ) that grazes the relativistic beam, and $\delta \approx \Gamma$. The fact that there are no sharp breaks in the light curve may indicate that the whole emitting area was visible, i.e., $\Gamma^{-1}>\theta_{\mathrm{j}}$. Therefore, we further assume a jet opening angle of a similar size to the relativistic beaming, say $\theta_{\mathrm{j}} \approx \Gamma^{-1} / 2$, and we get a jet power at the trigger time $(\Delta t=0) \quad$ of $\quad L_{\mathrm{j}}(\bar{\tau}) \approx 1.5 \times 10^{45} \mathrm{erg} \mathrm{s}^{-1}(\Gamma / 5)^{-4}$. Since $L_{\mathrm{j}}(\bar{\tau})=\epsilon_{\mathrm{j}} \dot{M}_{\mathrm{fb}}(\bar{\tau}) c^{2}$, it turns out that to have an efficiency $\epsilon_{\mathrm{j}}$ greater than $1 \%$ requires $m_{*} \leqslant 1 M_{\odot}$, for $\Gamma \leqslant 5$. In particular, $m_{*}=1 M_{\odot}$ gives efficiency between roughly $1 \%$ and $37 \%$ for $2 \leqslant \Gamma \leqslant 5$, which are in agreement with numerical simulations of jets from highly super-Eddingtonian accretion disks 
(Sądowski et al. 2014). Lower mass stars would give a higher efficiency range. We therefore assume in the following that $\mathrm{Sw}$ $\mathrm{J} 1644$ is the result of the disruption of a solar mass star. However, it is clear that this is just a tentative, though reasonable, choice, since the stellar mass cannot in fact be univocally determined, unless we can actually measure $\theta_{\mathrm{j}}$.

Assuming Sw J1644 as a prototype, we can adopt a general description of the X-ray light curve in the $1-10 \mathrm{keV}$ band, when we catch the flare after a time $\tau$ from the beginning of the event,

$$
L_{\mathrm{x}, \text { iso }}(\Delta t)=L_{\mathrm{x}, \mathrm{t}}\left(\frac{\tau+\Delta t}{\tau}\right)^{-5 / 3} .
$$

The (isotropic equivalent) luminosity $L_{\mathrm{x}, \mathrm{t}}$ at the time of the trigger $(\Delta t=0)$ is $L_{\mathrm{x}, \mathrm{t}}=L_{\mathrm{j}}(\bar{\tau}) \epsilon_{\mathrm{x}} \delta^{2} /\left(1-\cos \theta_{\mathrm{j}}\right) \simeq L_{\mathrm{j}}(\bar{\tau})$ $\epsilon_{\mathrm{x}} 2\left(\Gamma / \theta_{\mathrm{j}}\right)^{2}$, which can be written more explicitly as

$$
\begin{aligned}
L_{\mathrm{x}, \mathrm{t}} \approx & 1.63 \times 10^{48} \mathrm{erg} \mathrm{s}^{-1} M_{6}^{-1 / 2} m_{*, 1}^{1 / 2}\left(\frac{\epsilon_{\mathrm{x}}(z)}{0.2}\right) \\
& \times\left(\frac{t_{\min }+\bar{\tau}}{t_{\min }}\right)^{-5 / 3},
\end{aligned}
$$

where $\bar{\tau}=\tau /(1+z)$ and the radiation efficiency $\epsilon_{\mathrm{X}}(z)$ varies because of the spectral shifting with redshift,

$$
\epsilon_{\mathrm{x}}(z)=0.20 \frac{\left(E_{2}(1+z)\right)^{-\beta+2}-\left(E_{1}(1+z)\right)^{-\beta+2}}{\left(E_{2}\left(1+z_{\mathrm{sw}}\right)\right)^{-\beta+2}-\left(E_{1}\left(1+z_{\mathrm{sw}}\right)\right)^{-\beta+2}},
$$

where we assume $\beta=1.8, E_{1}=1 \mathrm{keV}, E_{2}=10 \mathrm{keV}$, and $\mathrm{z}_{\mathrm{sw}}=0.35$. We note that this correction is in the source rest frame and applies to unabsorbed fluxes.

In Equation (6), we set $\epsilon_{j} \Gamma^{2} / \theta_{\mathrm{j}}^{2} \approx 23.9$. Indeed, any combination of these quantities that gives a factor that allows us to reproduce the $\mathrm{Sw}$ J1644 X-ray luminosity at the trigger time. The degeneration should then be lifted, when we need to choose a Lorentz factor to compute the TDE rates. From the Xray luminosity, the flux is easily computed,

$$
F_{\nu}=\frac{L_{\mathrm{x}, \text { iso }}}{4 \pi D^{2}}
$$

where $D$ is the luminosity distance.

\subsection{Radio Lightcurve}

In this section, we first reproduce the light curve at $1.4 \mathrm{GHz}$ of Sw J1644 and then we generalize it to events at different redshifts and with different stellar and black hole masses.

The radio emission is synchrotron emission and the low energy spectrum can be described with the following broken power law:

$$
F_{\nu}=F_{\nu}\left(\nu_{\mathrm{a}}\right)\left[\left(\frac{\nu}{\nu_{\mathrm{a}}}\right)^{-2 s_{1}}+\left(\frac{\nu}{\nu_{\mathrm{a}}}\right)^{-s_{1} / 3}\right]^{-1 / s_{1}}\left[1+\left(\frac{\nu}{\nu_{\mathrm{m}}}\right)^{s_{2}}\right]^{-1 / s_{2}},
$$

(Granot \& Sari 2002) where $\nu_{\mathrm{a}}<\nu_{\mathrm{m}}$ are respectively the absorption and peak frequency; $s_{1}, s_{2}$ are smoothing factors; and the electron power-law index has been assumed to be 2.5 .

Berger et al. (2012) measure the flux $F_{\nu}\left(\nu_{\mathrm{a}}\right) \equiv F_{\nu, s w}\left(\nu_{\mathrm{a}, \mathrm{sw}}\right)$, and characteristic frequencies $\nu_{\mathrm{a}} \equiv \nu_{\mathrm{a}, \mathrm{sw}}$ and $\nu_{\mathrm{m}} \equiv \nu_{\mathrm{m}, \mathrm{sw}}$, in

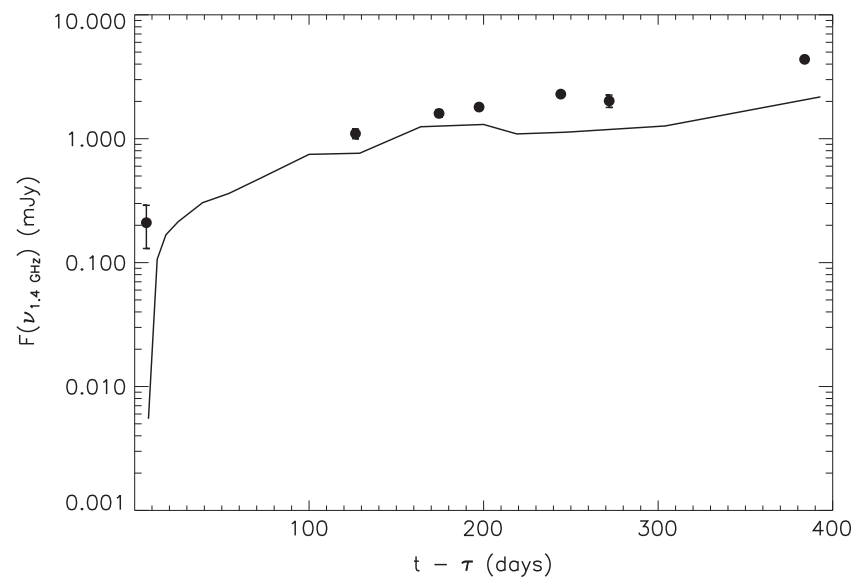

Figure 2. The radio $(1.4 \mathrm{GHz})$ light curve of $\mathrm{Sw} \mathrm{J} 1644$ as a function of time from the radio trigger ( 5 days after the X-ray first detection): data (circle marks) from Berger et al. (2012) and Zauderer et al. (2013) vs. our modeling (solid line). While our modeling well reproduces higher radio frequencies light curves (see Figure 1 in Berger et al. 2012), it slightly underpredicts the $1.4 \mathrm{GHz}$ one. In this respect our flux modeling is conservative.

several snapshots that cover the evolution of the light curve up to 220 days after the trigger. Later, Zauderer et al. (2013) extended the period of the radio monitoring up to $\sim 600$ days. The first observation is at $\sim 5$ days after the detection in the Xray band. Therefore, the radio emission is observed after a delay of $\tau \simeq 8$ days from the intrinsic beginning of the event. Finally, note that the radio data monitoring occurs up to $\sim 600$ days (Berger et al. 2012; Zauderer et al. 2013), while the X-ray emission has been observed up to $\sim 500$ days. This mismatch, however, is not a problem, since we are interested in modeling the light curves only up to one year after the explosion, when is already too dim to be detected by an X-ray survey in most cases.

Using the available data and Equation (8), we can therefore model the temporal evolution of the flux at any radio frequency. In Figure 2, we show the light curve of Sw J1644 at $1.4 \mathrm{GHz}$, and its comparison with data. A smooth temporal behavior has been obtained by linearly interpolating the flux between data points.

We now need to generalize our prototypical light curve to a generic TDE. The main uncertainty is how the flux scales with black hole and stellar masses. A first possibility is to describe the jet evolution with a Blandford Mckee (thereafter "BM model") solution, usually adopted for $\gamma$-ray burst afterglows (e.g., Berger et al. 2012; Metzger et al. 2012). Frequencies below $5 \mathrm{GHz}$ are in the self-absorbed part of the synchrotron spectrum, for the whole observed duration of the event (see Figure 3 in Berger et al. 2012). The observed specific luminosity in this regime $\left(\nu<\nu_{\mathrm{a}}<\nu_{\mathrm{m}}\right)$ is given by the Raleigh Jeans part of the blackbody spectrum $B(\nu / \delta) \delta^{3} \propto k_{\mathrm{b}} T(\nu / \delta)^{2} \delta^{3}$ (see Equation (8)), with a kinetic temperature given by $3 k_{\mathrm{b}} T=m_{\mathrm{e}} c^{2} \gamma_{\min }$, where the minimum Lorentz factor for the shocked accelerated electrons is $\gamma_{\min } \propto \Gamma$. Therefore the specific radio luminosity is

$$
L_{\nu} \propto B(\nu / \delta) \delta^{3}\left(r \theta_{\mathrm{j}}\right)^{2} \propto(r \Gamma)^{2}
$$

where $\left(r \theta_{\mathrm{j}}\right)^{2}$ is the emitting area, and we are assuming $\Gamma^{-1} \gtrsim \theta_{\mathrm{j}}$. In the blast wave modeling of J1644, the external 
medium swept up by the jet is better described by a power-law density decay that goes as $r^{-2}$, rather than a constant density environment (Zauderer et al. 2011). This implies $\Gamma \propto E_{\mathrm{j}}^{1 / 4}$ and $r \propto E_{\mathrm{j}}^{1 / 2}$, where $E_{\mathrm{j}} \approx L_{\mathrm{j}, \mathrm{p}} t_{\min } \propto m_{*}$ is the total jet energy. Therefore Equation (9) becomes $L_{\nu} \propto\left(L_{\mathrm{j}, \mathrm{p}} t_{\min }\right)^{3 / 2} \propto m_{*}^{3 / 2}$, where there is no dependence on the black hole mass, but only on the stellar mass.

The simple blast wave solution, however, does not describe the whole evolution of the radio spectrum (Berger et al. 2012). Therefore, we also consider a simpler approach. In line with our treatment of the X-ray flux, we may assume that the radio luminosity is proportional to the jet peak luminosity $L_{\nu} \propto L_{\mathrm{j}, \mathrm{p}} \propto M^{-1 / 2} m_{*}^{1 / 2}$, rather than to its total energy, (see the X-ray analog, Equation (6), which bears the same mass dependencies). As an extra motivation, this prescription may be justified in the context of the "magnetically arrested" jet model (e.g., Narayan et al. 2003). We will call this prescription "the Mass Dependent Luminosity" model (hereafter the MDL model).

The scaling of the peak flux for sources at different redshift, with different black hole and stellar masses (but at the same observed time $\tau$ from the beginning of the event) would be

$$
F_{\nu}\left(\nu_{\mathrm{a}}, \tau\right)=F_{\nu, \mathrm{sw}}\left(\nu_{\mathrm{a}, \mathrm{sw}}, \tau_{\mathrm{sw}}\right) \times m_{*, 1}^{3 / 2}\left(\frac{1+z}{1+z_{\mathrm{sw}}}\right)\left(\frac{D_{\mathrm{sw}}}{D}\right)^{2},
$$

for the $\mathrm{BM}$ solution and

$$
\begin{aligned}
F_{\nu}\left(\nu_{\mathrm{a}}, \tau\right)= & F_{\nu, \mathrm{sw}}\left(\nu_{\mathrm{a}, \mathrm{sw}}, \tau_{\mathrm{sw}}\right) \\
& \times M_{6}^{-1 / 2} m_{*, 1}^{1 / 2}\left(\frac{1+z}{1+z_{\mathrm{sw}}}\right)\left(\frac{D_{\mathrm{sw}}}{D}\right)^{2},
\end{aligned}
$$

for our second approach. The equivalent delay at which we need to calculate the flux of Sw J1644 is $\tau_{\mathrm{ws}} \equiv \tau \frac{1+z_{\mathrm{sw}}}{(1+z)}$.

The characteristic frequencies need to be redshifted ${ }^{4}$ according to

$$
\nu_{\mathrm{a}}(\tau)=\nu_{\mathrm{a}, \mathrm{sw}}\left(\tau_{\mathrm{sw}}\right)\left(\frac{1+z}{1+z_{\mathrm{sw}}}\right)^{-1},
$$

and

$$
\nu_{\mathrm{m}}(\tau)=\nu_{\mathrm{m}, \mathrm{sw}}\left(\tau_{\mathrm{sw}}\right)\left(\frac{1+z}{1+z_{\mathrm{sw}}}\right)^{-1} .
$$

In all cases, the flux $F_{\nu, \mathrm{sw}}\left(\nu_{\mathrm{a}, \mathrm{sw}}, \tau_{\mathrm{sw}}\right)$ and the characteristic frequencies at any time $\tau_{\mathrm{sw}}$ are obtained by linearly interpolating the available data. For $\tau_{\mathrm{sw}}<8$ days we extrapolate the radio light curve to earlier epochs.

\section{BLACK HOLE MASS FUNCTIONS}

The mass distribution of black holes as a function of redshift is an essential ingredient to calculate TDE rates. Since black holes grow mainly by efficient accretion (Soltan 1982), one can calculate these functions using the

\footnotetext{
4 Formally, one would need to consider the transformation due to different Doppler factors between jets. However, we here assume that all jets have approximately the same Lorentz factor $\Gamma$ and viewing angle of nearly $\theta_{\mathrm{o}} \approx 1 / \Gamma$. The latter is because the viewing angle probability $\left(\propto \theta_{0}\right.$, between $\left.0<\theta_{0}<\Gamma^{-1}\right)$ is the highest at $\Gamma^{-1}$.
}
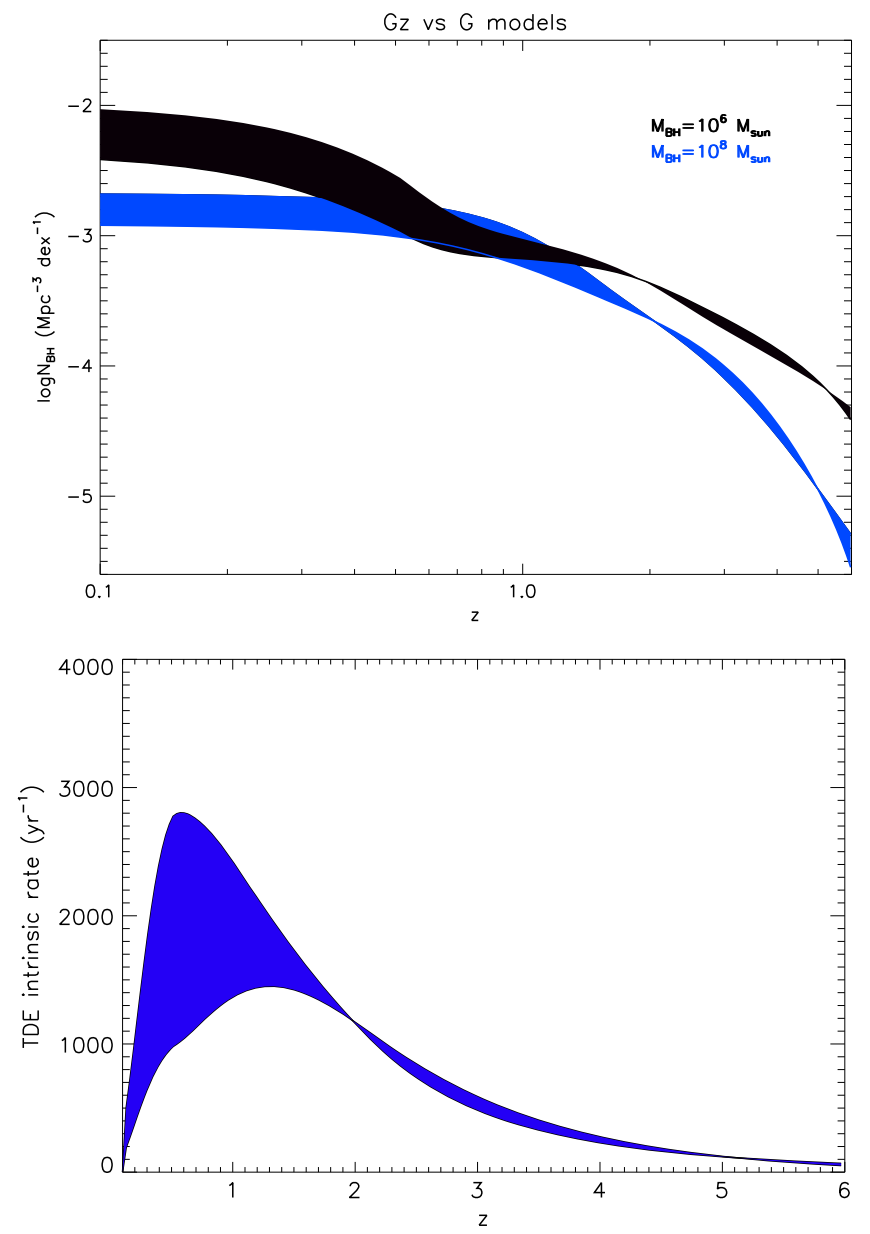

Figure 3. Upper panel: BH number density as a function of $z$ for $10^{6}$ (black shaded area) and $10^{8} M_{\odot}$ (blue shaded area). Lower panel: intrinsic rate of TDEs as a function of redshift. A rate of $10^{-5} \mathrm{yr}^{-1}$ per galaxy is assumed. Most of the events are expected below $z \sim 2$.

mass continuity equation, given a radiation efficiency and distribution of Eddington ratios. In this paper, we use the results from Shankar et al. (2013). In particular, we consider the two accretion models that yield the largest and the lowest black hole comoving number density $\phi(M, z)$, and are still consistent with the quasar bolometric luminosity functions and the local black hole mass function (models labeled $G$ and $G(z)$ in Shankar et al. 2013). In this way, we can estimate the uncertainty due to the black hole mass distribution of our expected TDE rates. In Figure 3 upper panel, we show the mass distribution functions and their uncertainty strips as a function of redshift, for $M=10^{6} M_{\odot}$ and $M=10^{8} M_{\odot}$ black holes. In Figure 3, instead, we show the "intrinsic" TDE rate as a function of redshift,

$$
R(z)=\int_{M_{\min }}^{M_{\max }} \phi(M, z) V(z) N_{\mathrm{tde}} d M,
$$

where we denote with $V(z)$ the comoving cosmological volume. $N_{\mathrm{tde}}=10^{-5} \mathrm{yr}^{-1}$ is our fiducial TDE rate per galaxy: this value is in the range of theoretical expectations (Merritt 2013) and observational claims (Donley et al. 2002; Gezari et al. 2009; van Velzen et al. 2011). 
The minimum black hole mass (here and thereafter in our calculations) is $M_{\min }=10^{6} M_{\odot}$, as just a few SMBHs have been observed with a lower mass. ${ }^{5}$

\section{MONTE CARLO CALCULATIONS}

Assuming the X-ray and radio modeling described in Section 2 we perform Monte Carlo simulations (MCs) to derive the number of jetted TDEs to be detected per year, for the given flux limit and sky coverage.

Beside the $\mathrm{BH}$ mass, the main ingredients of our MCs are the trigger lag time, $\tau$, and the mass of the disrupted stars, $m_{*}$. The former is randomly extracted from a uniform distribution between 0 and 1 yr. $^{6}$ The latter follows a Kroupa Initial Mass Function (IMF; Kroupa 2001),

$$
f(m) \propto \begin{cases}m^{-0.3}, & 0.01 \leqslant m_{*} \leqslant 0.08 \\ m^{-1.3}, & 0.08 \leqslant m_{*} \leqslant 0.5 \\ m^{-2.3}, & m_{*}>0.5\end{cases}
$$

In fact, for each black hole mass, the minimum stellar mass is set by the requirement that the tidal radius should be greater than the last stable orbit (we assume a non-spinning $\mathrm{BH}$ ). This requirement implies that $m_{* \min }=\max \left[0.01,0.045 M_{6}\right]$. Note that for $M=10^{8} M_{\odot}$, the minimum mass is $m_{* \min }=4.5 M_{\odot}$. Therefore, events associated with high $\mathrm{BH}$ masses are suppressed in numbers by the steepness of the IMF, as only $0.4 \%$ of all stars have $m_{*}>8$. However, they are on average brighter because the average $m_{*}$ is larger.

In our simulation, we start by considering the intrinsic rate $R$ (z) (Equation (12)) properly modified by accounting for the relativistic beaming, which results in a reduction by a factor of $2 \pi \Gamma^{-2} /(4 \pi)=1 /\left(2 \Gamma^{2}\right)$ : this is the fraction of solid angle subtended by the emission, when considering a two sided jet. Our fiducial value for the jet Lorentz factor is $\Gamma=2$, as inferred by radio observations $(\Gamma \approx 2$, Berger et al. 2012; Zauderer et al. 2013). If the jet decelerates, this value has to be intended as an average one, over the observation period. However, we note that this is a geometrical scaling factor and our results may be easily re-scaled by assuming different values of the jet bulk Lorentz factor. In addition, $R(z)$ is scaled for the fraction of the sky surveyed by the assumed instrument. In the calculation of $R(z)$ we have adopted both $G$ and $G(z)$ models in order to account for the systematic uncertainties in the mass function modelings. The number of trials in MCs is properly fixed by requiring a high statistics level in each mass and redshift bin (typically $\geqslant 10^{4}$ ).

\section{RESULTS}

In this section, we first validate separately our emission models for X-ray and radio light curves by comparing our predicted rates with current instruments and survey results. In fact, we find that current data do not put strong constraints on our modeling, as we will explain in the following. Future data

\footnotetext{
The recent discovery of TDEs in dwarf galaxies $\left(\lesssim 10^{6} M_{\odot}\right)$ (Donato et al. 2014; Maksym et al. 2014b, 2014a) seems particularly promising in overcoming this limit and use TDEs to find lower mass BHs.

6 We do not use longer time lags because any extrapolation beyond the currently available radio data would make our estimates more modeldependent, since there is no hydrodynamical model that can reproduce the whole radio behavior of $\mathrm{J} 1644$.
}

have instead a greater potential. In the SKA era, we propose that a strategy where radio will be triggering X-ray facilities can allow us not only to detect but also to identify and investigate jetted TDEs in a multi-wavelength fashion. In the following, if not otherwise mentioned, our results are derived adopting $\Gamma=2$.

\subsection{Comparison with Current Surveys \\ 5.1.1. Hard X-rays}

So far, only two jetted TDE candidates have been detected by BAT, implying an observed rate of $\sim 0.3 \mathrm{yr}^{-1}$.

Since BAT is not operating in survey mode, it is not straightforward to compare observations with our predictions, i.e., it is difficult to choose sky coverage and detection limit, because they are not univocally determined. The two TDE candidates were detected in two different modes: Sw J1644 was triggered on board, while Sw J2058 was discovered by stacking four-day integration images (Krimm et al. 2011; Cenko et al. 2012). In both modes, it is hard to define a survey flux limit, the key ingredient of our MCs. Indeed, Swift has over 500 onboard trigger criteria in different modes which makes the use of a flux limit survey a rather simplified approach. The same applies to possible discoveries of fainter TDEs with longer integration times, by applying the image mosaics technique (Krimm et al. 2013). These have to be promptly followed-up by XRT for their identification: monitor the soft $\mathrm{X}$-ray emission and then measure the characteristic temporal slope of TDEs. In this way, a further efficiency accounting for any reason preventing XRT to monitor the event has to be included in our rate calculations (e.g., the stochastic nature of the Swift pointing plan, the target visibility and the mission schedule; H. Krimm, private communication). Such an efficiency is hard to quantify and any assumption would be arbitrary and would bias our discussion on the comparison between the predicted and observed rates. In addition to that, our soft X-ray modeling assumes a total disruption of the star (see Section 2), while the Sw J2058 emission seems to be consistent with a partial disruption. For both of these reasons, we focus on detections triggered onboard, although with due caveats. In fact, any reliable prediction based on onboard triggers would require complex simulations as done by (Lien et al. 2014) for GRB rates. We therefore set to achieve a less ambitious aim at predicting indicative rates, which should be considered most likely as upper limits. Specifically, we adopt the BAT daily sky coverage reported in Krimm et al. (2013) and fix a unique "survey" flux limit to be consistent with the detection of the Sw J1644. We detail the procedure in the following.

Sw J1644 was detected with an onboard BAT image trigger (Cummings et al. 2011). In this trigger mode, we assume a flux limit of $2.5 \times 10^{-9} \mathrm{erg} \mathrm{cm}^{-2} \mathrm{~s}^{-1}$ in the $15-150 \mathrm{keV}$ band, which is consistent with the faint tail of the observed GRB rate (Lien et al. 2014) and the detection of Sw J1644 (Burrows et al. 2011). We adopt a daily sky coverage of $85 \%$ (Krimm et al. 2013) and apply an efficiency of $\sim 90 \%$, for the fraction of the BAT survey time (Lien et al. 2014) resulting from trigger deadtimes (e.g., due the passage through the South Atlantic Anomaly).

The detection rate is estimated by performing a large set of MCs as described in Sections 2.2 and 4. In Equation (5), we use a radiation efficiency of $\sim 0.3$ (Burrows et al. 2011). For 
Table 1

Future Radio and X-ray Surveys Predictions

\begin{tabular}{|c|c|c|c|c|c|c|}
\hline & $\begin{array}{c}R^{1} \\
\left(\mathrm{yr}^{-1}\right)\end{array}$ & $\begin{array}{c}R^{2} \\
\left(\mathrm{yr}^{-1}\right)\end{array}$ & $z_{\text {peak }}$ & $\begin{array}{l}R_{\text {peak }}^{1} \\
\left(\mathrm{yr}^{-1}\right)\end{array}$ & $\begin{array}{l}R_{\text {peak }}^{2} \\
\left(\mathrm{yr}^{-1}\right)\end{array}$ & $z_{\max }$ \\
\hline \multicolumn{7}{|c|}{ Radio Selected Sample } \\
\hline SKA $M D L$ & 327 & 770 & 0.4 & 14 & 40 & 1.7 \\
\hline$L O F T$-like $B M$ & $128(2.5)$ & $305(6.5)$ & 0.3 & $4.5(0.05)$ & $13(0.1)$ & 1.7 \\
\hline$L O F T$-like $M D L$ & $135(1.3)$ & $352(3.5)$ & 0.4 & $8(0.08)$ & $22(0.2)$ & 1.2 \\
\hline \multicolumn{7}{|c|}{ X-ray surveys } \\
\hline $\mathrm{BAT}^{3}$ & $9.5(0.095)$ & $26.5(0.26)$ & $0.1-0.2$ & $1.7(0.02)$ & $4.6(0.05)$ & 0.32 \\
\hline eRosita & $8(0.08)$ & $15(0.15)$ & 0.4 & $0.15(0.001)$ & $0.5(0.005)$ & 0.4 \\
\hline Einstein Probe & $89(0.9)$ & $242(2.4)$ & 0.3 & $5.5(0.05)$ & $15(0.2)$ & 1 \\
\hline LOFT-like WFM & $24.5(0.2)$ & $67(0.7)$ & 0.2 & $2.3(0.02)$ & $6(0.06)$ & 0.6 \\
\hline
\end{tabular}

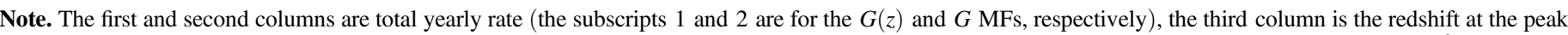

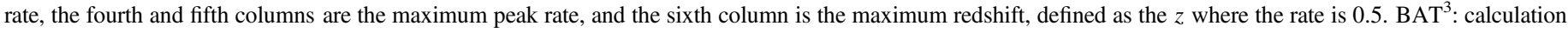
for an onboard image trigger. X-ray and radio expected rates are derived for $\Gamma=2$. X-ray rates are also reported for $\Gamma=20$ in parentheses.

each event, we compare the flux at the trigger time $\tau$ with our flux threshold. We obtain a TDE rate of $\approx 10-20$ events $\mathrm{yr}^{-1}$ (see Table 1). The rate distribution with redshift extends up to $z_{\max } \approx 0.32$ and peaks ${ }^{7}$ at $z \approx 0.2$. The peak value ranges between 1-5 $\mathrm{yr}^{-1}$ (see Table 1). The peak of the corresponding $\mathrm{BH}$ mass distribution is at $10^{6} M_{\odot}$ and contains $\sim 23 \%$ of all events.

Given our predicted mass and $z$ distributions for the observed TDEs, an event like Sw J1644 has a chance probability that is a factor of $\sim 10$ lower than that of an event at the peak rate. Therefore, it is not an unlikely event, but a lower redshift object would have had a higher probability. At this point, it is unclear to us if this result is more due to our simplified treatment of the BAT trigger or to our assumption of a constant jet luminosity for a given $\mathrm{BH}$ and stellar mass. Both are very likely to have a role. But since we cannot trust at this level our trigger modeling and the paucity of detected events does not constrain a possible luminosity function, we do not attempt here to modify our X-ray model to fit this observed distribution. When more events will be identified, our procedure can be refined to account for a TDE variety. A comparison with future, easier to model, surveys (see Section 5.2.1) will also help in constructing a more robust emission model.

Interestingly, these rates are actually up to two orders of magnitude higher than that $\left(0.3 \mathrm{yr}^{-1}\right)$ derived from BAT observations, but a key role is played by the low value of the $\Gamma$ considered. We will elaborate on this point in Section 6.1.

\subsubsection{Radio Surveys at $1.4 \mathrm{GHz}$}

We compare our predictions with constraints on the jetted TDE rate derived from current radio surveys (Bower 2011). In the following radio estimates, we will require a $5 \sigma$ flux limit to claim detection.

We first consider the combined catalogs of VLA First and NVSS at $1.4 \mathrm{GHz}$. The combined sky coverage is $0.19 \mathrm{sr}$ with a

\footnotetext{
7 Here and in the following, we define $z_{\max }$ as the redshift at which the expected rate is $0.5 \mathrm{yr}^{-1}$.
}

flux limit of $6 \mathrm{mJy}$ at $1.4 \mathrm{GHz}$. The analysis of these catalogs did not yield any TDE candidate.

To derive our predictions, we adopt the radio modeling described in Section 2.3, and for each event (i.e., for each set of $\tau$, black hole and stellar mass, and redshift), we calculate the average flux over a period of one day from the trigger. This is compared with a $6 \mathrm{mJy}$ flux threshold. Rescaling our all-sky results for the catalog sky coverage, we obtain an observed rate that even in the most favorable case $\left(\sim 0.3 \mathrm{yr}^{-1}\right.$ using BM model, Equation (10)) is consistent with the Bower (2011) and Frail et al. (2012) results. To strengthen this conclusion, we note that our assumption of a $6 \mathrm{mJy}$ threshold per day combined with a sky coverage of 0.19 sr may be considered already rather optimistic, since both values are referring to a one-year single epoch.

In the near future, the VLA Stripe 82 survey may constrain jetted TDE models thanks to the improved sensitivity $(50 \mu \mathrm{Jy}$ rms) at $1.4 \mathrm{GHz}$ over a FoV of $90 \mathrm{deg}^{2}$ (Hodge et al. 2013). By assuming a $5 \sigma$ threshold of $0.25 \mathrm{mJy}$, our modeling predicts a number of a few objects to be detected per year. Significant advances in TDEs detections are expected to come from ongoing wide radio surveys at both low (see, e.g., MWA and LOFAR) and high radio frequencies (e.g., ASKAP and VLASS). Since our radio modeling was constrained by observations at higher $(>1.4 \mathrm{GHz})$ radio frequencies (as discussed in Section 2.3), we focus here on ASKAP and VLASS. The Variable and Slow Transient (VAST) project on ASKAP envisages a sky coverage of $10^{4} \mathrm{deg}^{2}$ reaching a sensitivity of $0.5 \mathrm{mJy}$ rms (VAST wide) with a daily cadence (Murphy et al. 2013). Our predictions for VAST are in the range of a few up to $\sim 14$ TDEs $\mathrm{yr}^{-1}$, consistent with expectations from Murphy et al. (2013). For comparison, Frail et al. (2012) obtain a value of $\sim 82 \mathrm{yr}^{-1}$ by considering longer integration time ( $\sim 10$ days). In the case of the VLA Sky Survey (VLASS; Hallinan et al. 2013), we consider a sky coverage of $10^{3} \mathrm{deg}^{2}$ with $\sim 3$ week cadence at a sensitivity of $0.1 \mathrm{mJy}$ rms. This setup should give a number between two and six objects to be detected per year. All-sky VLASS is also foreseen and will clearly provide a larger number of 
TDEs, but we focus on the previous strategy because the multiepoch survey could provide alerts for follow up at higher energies, with a prompt identification of the transient.

\subsection{Future Instruments}

Currently, the only two jetted TDE candidates were discovered in X-rays, where the characteristic $t^{-5 / 3}$ decay slope has been observed. Therefore, we first discuss the discovery potential of future X-ray surveys. We then predict the expected rate of TDEs for the SKA $1.4 \mathrm{GHz}$ wide survey. Finally, we derive the properties and rate of TDEs that can be detected in radio with SKA and subsequently identified in X-rays.

\subsubsection{Future X-ray Surveys}

The rate estimates provided in this section are based on a unique observing strategy aimed at detecting and providing a first identification of the transient as a TDE. We assume that a given fraction of the sky is covered in one day at a flux threshold defined by the requirement to follow the typical TDE decay over four light-curve bins, each with signal-to-noise ratio $(\mathrm{S} / \mathrm{N}) \geqslant 5$. This is obtained by starting from the $5 \sigma$ flux limit of each survey, then tracing back the $t^{-5 / 3}$ decay in order to obtain the flux over the four time bins and then computing the average flux over that period. This average flux defines the identification flux threshold. We will give values for both $\Gamma=2$ and $\Gamma=20$ and we will justify this choice and elaborate on the comparison in Section 6.

The all-sky survey mission eRosita (Merloni et al. 2012) is expected to detect jetted TDEs, in its "hard" X-ray band $(2-10 \mathrm{keV})$. We apply our methodology to the eRosita survey, properly re-scaling the sky coverage achieved in a six-month scan to one day. We derive the identification flux threshold for our observing strategy from the $2-10 \mathrm{keV} 5 \sigma$ sensitivity of $\sim 10^{-12} \mathrm{erg} \mathrm{cm}^{-2} \mathrm{~s}^{-1}$, corresponding to $\sim 250 \mathrm{~s}$ exposure (Merloni et al. 2012) as foreseen for each point in the sky. We calculate the corresponding unabsorbed flux and then we extrapolate it in the energy range $1-10 \mathrm{keV}$ (used in our X-ray modeling). We predict a maximum of $\sim 15$ TDEs per year to be detected up to $z \approx 2.5$, although $z_{\max } \approx 0.4$. The peak rate is between 0.15 and $0.5 \mathrm{yr}^{-1}$ at $z \approx 0.4$ and beyond $z \approx 2$, the rate is $<5 \times 10^{-2} \mathrm{yr}^{-1}$. If a larger value of $\Gamma=20$ is considered, the rate decreases by two orders of magnitude (see Table 1) with a maximum total rate of $\sim 0.15 \mathrm{yr}^{-1}$ and peak rate of only $4 \times 10^{-3} \mathrm{yr}^{-1}$. We therefore predict both higher $(\Gamma=2)$ and lower $(\Gamma=20)$ rates than those previously published by Khabibullin et al. (2014; one object to be detected per six-month-long scan), but we definitively reach a much lower redshift $\left(z_{\max }=0.4\right.$ versus their $\left.z=4.5\right)$. The same authors provide an upper limit of $\approx 150$ events per scan by considering the number of jetted TDEs to be one-fifth of their "soft" TDE sample $(\approx 1000)$. This fraction is based on results from the ROSÁT X-ray survey (Donley et al. 2002). We are clearly consistent with their estimate.

The Wide Field Monitor (WFM) on board a LOFT-like (Feroci et al. 2012) mission will also have the capability to trigger jetted TDEs by surveying one-third of the sky with a $5 \sigma$ one-day sensitivity of $\sim 8-9 \times 10^{-11} \mathrm{erg} \mathrm{cm}^{-2} \mathrm{~s}^{-1}$ (a few $\mathrm{mCrab}$ ) in $2-50 \mathrm{keV}$ energy band. We estimate tens of objects per year up to $z_{\max } \approx 0.6$. The peak rate is $\sim 6 \mathrm{yr}^{-1}$ at $z=0.2$.
These numbers imply a total rate of $0.7 \mathrm{yr}^{-1}$ for $\Gamma=20$ with a peak rate of only $6 \times 10^{-2} \mathrm{yr}^{-1}$.

Finally, we consider the Einstein Probe, which is expected to monitor half of the entire sky in the energy range $0.5-4 \mathrm{keV}$ with a $5 \sigma$ sensitivity of $\sim 10^{-11} \mathrm{erg} \mathrm{cm}^{-2} \mathrm{~s}^{-1}$ in each point $(1 \mathrm{ks}$ exposed) of the sky (EP; W. Yuan 2013, private communication). In this case, MCs were adapted in order to extend our Xray modeling to this energy range. This requires to first estimate the unabsorbed flux limit by accounting for both the Galactic and the intrinsic absorption (Burrows et al. 2011) and then calculate a proper radiation efficiency by extrapolating from the value inferred in $1-10 \mathrm{keV}$. We estimate a number between $\sim 90-240 \mathrm{yr}^{-1}$ to be detected below $z_{\max } \approx 1$ with a peak rate of $\approx 15 \mathrm{yr}^{-1}$ at $z=0.3$. In the case of $\Gamma=20$, a few objects are expected to be detected per year, with a peak rate of $\sim 0.2 \mathrm{yr}^{-1}$. A summary of the actual numbers can be found in Table 1.

Inspecting the trigger time distributions (see top panel in Figure 5), we find that up to $z_{\max } \sim 1$ objects are detected with almost equal probability at any delay from the explosion.

These X-ray survey rates have been obtained under the assumption of a reasonable observing strategy. A larger sample extending up to higher redshift can be obtained if longer integration times are considered, but these predictions are affected by several parameters like the trade-off between sky coverage and sensitivity. In this respect, our approach has to be considered conservative.

\subsubsection{SKA as a TDE Hunter}

Presently, the most ambitious and revolutionary project in radio astronomy is the Square Kilometer Array (SKA; Carilli \& Rawlings 2004) planned to begin operating in 2020. SKA, in survey mode (SKA1-Survey; Dewdney et al. 2013), is able to achieve a half-sky coverage $\left(20,000 \mathrm{deg}^{2}\right)$ with two-days cadence at a $5 \sigma$ flux limit of $90 \mu \mathrm{Jy}$ (Donnarumma et al. 2014; Feretti et al. 2014). This unprecedented sky coverage and sensitivity make SKA an optimal radio transient hunter.

In contrast to X-ray searches, in radio, we cannot have a first identification based on the light curve, since the $1.4 \mathrm{GHz}$ radio emission of a TDE is not particularly different from those of other radio transients (e.g., GRB, blazars). Therefore, we consider a different strategy. In our MC simulations, we directly assume the SKA $5 \sigma$ flux limit in order to claim the detection of a transient event. The identification strategy will fully rely on the multi-frequency follow-up of the trigger event as it will be discussed at the end of this section.

We calculate the predicted average flux over two days from the trigger and then we compare it with the SKA flux limit. The results are shown in Figure 4. The upper panels are derived using the BM model (Equation (10)) for the radio light-curve modeling, while the lower panels use the MDL model (Equation (11)). There, we show the distribution of the TDE rate as a function of $z$ (right panels) and their $\mathrm{BH}$ mass distribution (left panels) for the two $\mathrm{BH}$ mass functions described in Section 4 (black lines). The yellow lines show the subclass of events with BH masses lower than $10^{7} M_{\odot}$. Both radio models produce redshift distributions peaking around $z \approx 0.4$, regardless of the $\mathrm{BH}$ mass function. The peak rates are roughly between 6 and $40 \mathrm{yr}^{-1}$ (see also Table 1). Events with $\mathrm{BH}$ mass lower than $10^{7} M_{\odot}$ dominate the distributions at all redshifts in the MDL model, while this only happens at $z<0.4$ in the BM model. One marked difference between the two 

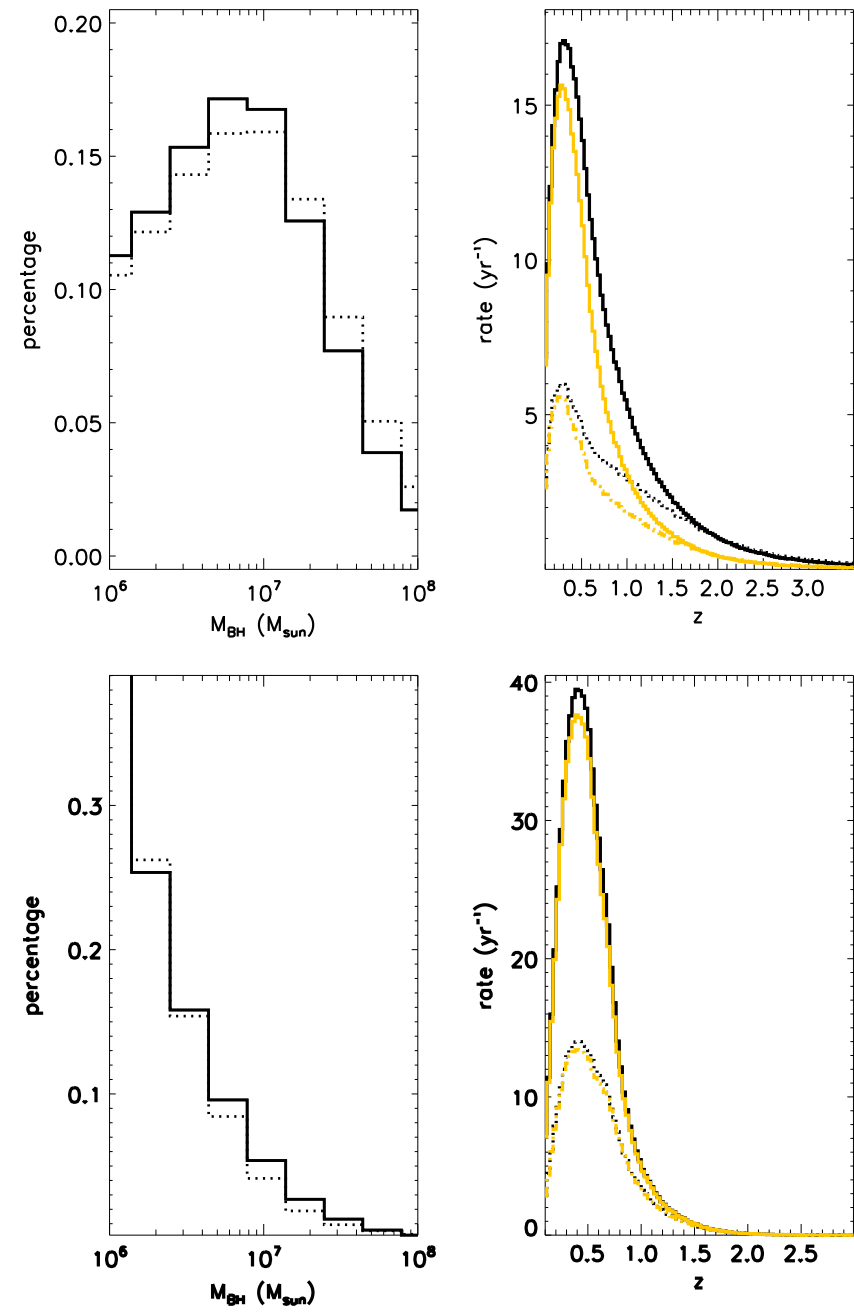

Figure 4. Rate of events predicted for SKA in wide survey mode at $1.4 \mathrm{GHz}$ as a function of redshift (right panels) and their distribution as a function of $\mathrm{BH}$ mass (left panels), for two different black hole distribution functions (black solid line: $G$ model, black dotted line: $G(z))$ model. Rates associated with events with $\mathrm{BH}$ masses lower than $10^{7} M_{\odot}$ are also shown (yellow lines). Upper panels: BM model for the jet evolution; lower panels: MDL model.

radio light-curve modelings is the $\mathrm{BH}$ mass distribution of the detected events: while BHs with masses between $10^{7}-10^{8} M_{\odot}$ are equally probable in the BM model (because the flux is $\mathrm{BH}$ mass independent, Equation (10)), BHs with mass $<3 \times 10^{6}$ completely dominate the observed sample in the MDL model. As a consequence, the BM model distribution extends to higher redshifts $\left(z_{\max } \approx 2.5\right.$ versus $\left.z_{\max } \approx 1.7\right)$ because $\mathrm{BH}$ masses larger than $10^{7} M_{\odot}$ interact with higher mass stars (higher $\left.m_{*, \min }\right)$ and produce intrinsically brighter flares. This will allow us to study TDEs close to the peak of cosmic star formation.

In Table 1, we also report the total rates obtained by integrating these distributions in $z$ and $M_{\mathrm{BH}}$. We obtain yearly rates of the order of a few to several hundred. These results are not consistent with those that can be derived by using Equation 4 in van Velzen et al. (2013): inserting our SKA survey parameters, we obtain thousands of events per year. This discrepancy is due to our inclusion of the stellar mass dependence, that modulates the TDE luminosity for a given BH mass: the lower the $m_{*}$, the dimmer the event. In the assumption of a Kroupa IMF, the bulk of the events are caused by the disruption of stars with $m_{*}<1$, increasing the number of flares that are too dim to be detected.

With hundreds of events per year, SKA could be able to detect more TDEs than any currently planned X-ray survey. On the other hand, while X-ray surveys can catch the events soon after explosion (see Einstein Probe performance in Figure 5, upper panel, for an example), SKA would not be able to cover the first week activity at any redshift and only at $z<0.8$ will SKA probe the first month (Figure 5, bottom panel). This result is independent of the assumed radio modeling. The explanation is simple: the observed radio flux at $1.4 \mathrm{GHz}$ is initially increasing, contrary to that in the X-ray band. In this regard, detections in these two bands are complementary. However, a word of caution here is due. As mentioned before, below 10 days, we have virtually any detection in radio at any $z$. This early period coincides with the rise of the radio light curve. Although we expect this gap in detection, the exact epoch at which it occurs depends on the detailed behavior of the light curve during this undetected rise. Our extrapolation at earlier times is quite steep and we consider 10 days as an upper limit for the initial gap in detection.

Thus far, we have focused on the detection of TDEs with SKA, which, depending on the observing strategy, will only be a fraction of a noticeable sample of slow radio transients. As mentioned earlier, we cannot use radio properties or variability alone to distinguish a TDE candidate from either a slowly variable AGN or a GRB. A possibility of identification that we explore below is through quick follow-ups at higher energies, particularly in X-rays. A first pre-screening of the radio candidates could be done by cross-correlating the radio transient positions with deep AGN catalogs, expected to be provided in the near future by optical surveys (e.g., LSST) or the SKA precursors (e.g., ASKAP). However, we expect a larger degree of contamination of the TDE sample to come from transient sources such as GRBs. Since, unlike GRBs, most TDEs should have a nuclear origin, it is mandatory to quickly identify the host galaxy. An accurate localization of the radio transient in the core of galactic nuclei, helping to assess the nuclear origin, will therefore play a major role in the screening of the radio transient sample. This means that first the host galaxy has to be found by a rapid optical follow-up and after the brighter transients could be localized by SKA with a precision of $\sim 100$ mas essential ${ }^{8}$ to separate nuclear transients from other phenomena (e.g., GRB).

For details see Donnarumma et al. 2014.

\subsubsection{Combining Radio and X-rays in the SKA Era}

$\mathrm{X}$-ray follow-up will have a major role in the identification of the TDE candidate detected by SKA because of the possibility to detect the characteristic $t^{-5 / 3}$ decay. A possible X-ray follow-up strategy aimed at identifying and then characterizing the event consists in a fast repointing of the transient detected by SKA. We consider a one-day delay in the $\mathrm{X}$-ray repointing and require a set of X-ray observations spread over a few days in order to follow the characteristic temporal decay of the TDE. We foresee an observing strategy that is similar to the one adopted in the case of future X-ray surveys (see Section 5.2.1): four observations spread over four days, with $\mathrm{S} / \mathrm{N}$ ratio $\geqslant 10$ in each. A high $\mathrm{S} / \mathrm{N}$ is required in order to

\footnotetext{
8 This can be achieved thanks to the resolution of about 2 arcsec of SKA1SUR and 0.6 arcsec or better of SKA1-MID (Dewdney et al. 2013).
} 

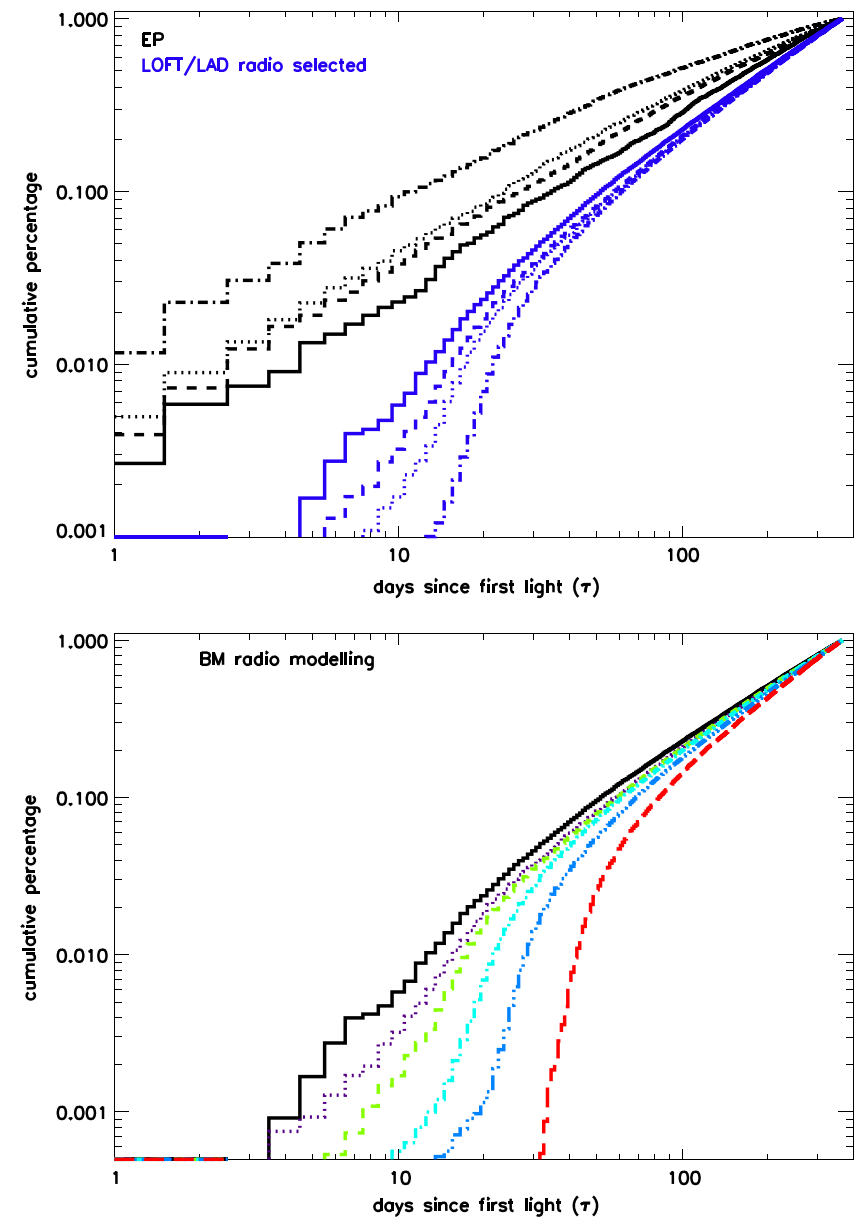

Figure 5. Cumulative distributions of delays in detecting the TDE from the explosion time, for different redshifts. Top panel: Einstein Probe (black lines) and LAD follow-ups of radio triggered TDEs (blue lines). The different line styles are for $z=0.1,0.2,0.37$, and 0.8 from right to left for Einstein Probe and vice versa for LAD. Bottom panel: the same as above but for SKA $\mathrm{BM}$ model and $z=0.1,0.2,0.37,0.8,1.5$, and 3 from left to right).

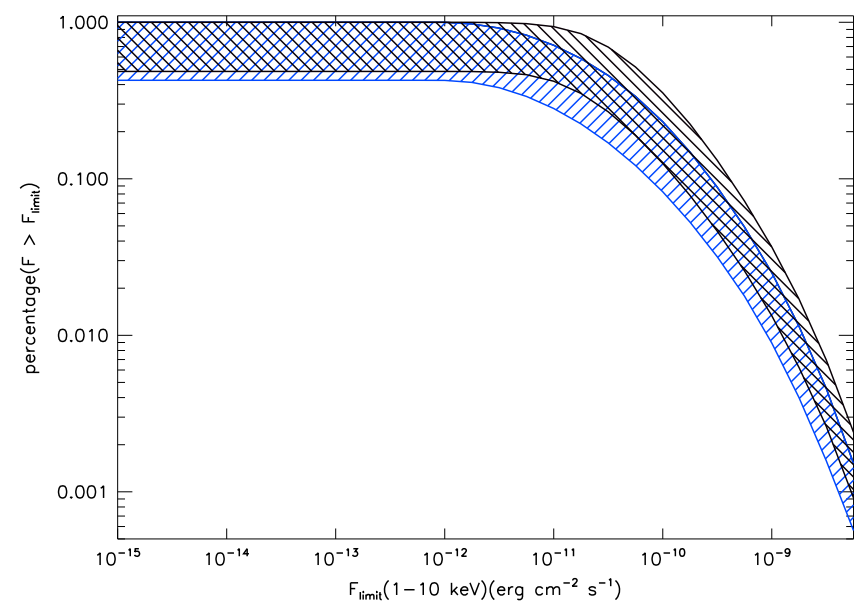

Figure 6. Fraction of X-ray TDEs that can be identified, following up a SKA trigger, as a function of flux limit (unabsorbed flux). The blue and black shaded area are obtained with Equations (10) and (11) radio modelings, respectively. The shaded areas reflect uncertainties in $\mathrm{BH}$ mass functions. The figure shows that a X-ray instrument with a flux limit of $\lesssim 10^{-11}$ in cgs units, can in principle identify any radio detected TDE. See the text for details. characterize both the temporal and spectral behavior of the source.

For each event in the MCs, we calculate the average X-ray flux over the four days after the repointing and compare it with the identification flux threshold derived as explained in Section 5.2.1, with the only difference being an $\mathrm{S} / \mathrm{N} \geqslant 10$ requested in each observation. Practically, $\tau$ in Equation (5) has to be the radio trigger time-lag, plus an extra delay of one day for repointing, and $0 \leqslant \Delta t \leqslant 3$. In this way, we derive the properties of samples of TDEs, which are first detected in radio and promptly followed-up in X-rays.

In Figure 6, we show the fraction of SKA candidates that can be identified as a function of the X-ray (1-10 keV) unabsorbed flux limit. A rapid X-ray follow-up will be able to detect a complete radio-selected sample provided that the instrument sensitivity is close to $F_{\text {lim }} \lesssim 10^{-11} \mathrm{erg} \mathrm{cm}^{-2} \mathrm{~s}^{-1}$ in the energy. In fact, a moderate sensitivity $\sim 10^{-11}-10^{-10} \mathrm{erg} \mathrm{cm}^{-2} \mathrm{~s}^{-1}$ is already enough to detect equal or a larger number of events than with X-ray wide sky instruments alone. It is therefore clear that a radio trigger is a more efficient way to build up a large $\mathrm{X}$ ray sample of TDEs. Rates reported in that figure assume a fast (one day) X-ray repointing and $F_{\text {lim }}$ reached with an integration of similar to four days. Rates could be substantially different if longer integrations are needed to reach the same $F_{\text {lim }}$ or in the case of longer repointing time. This is a natural consequence of the decreasing trend of the X-ray light curve.

When considering an actual follow-up strategy, the values reported in Figure 6 should be scaled by the fraction of sky accessible to the X-ray instrument considered. In general, the $\mathrm{X}$-ray follow-up will provide us with a sub-sample of radio triggered TDEs, defined by the target accessibility, the repointing chance of the X-ray satellite and the sensitivity of the instruments. Since TDEs also emit in hard X-rays, a tradeoff between sensitivity, sky coverage, and a broad energy range is foreseen; in particular, the broader the energy range, the better the characterization of the non-thermal process and of the jet energy budget.

Future X-ray experiments like Athena (Nandra et al. 2013) and a LOFT-like mission (Feroci et al. 2012) could offer a unique chance to follow-up and characterize SKA-triggered TDEs. Moreover, if Swift were still operating in the 2020s, XRT will have great potential to follow up the radio candidates.

Athena's sensitivity lies in the saturation branch of Figure 6, which implies that the observed rate of X-ray jetted TDEs will be crucially linked to its follow-up efficiency. This is mainly influenced by the Athena sky accessibility, which is of the order of $\sim 50 \%$ (Athena mission proposal), resulting in a rate of TDEs of a few hundred, with detections up to $z_{\max } \approx 2$ (see Table 1 ).

The LAD (Large Area Detector) on board of LOFT $(2-50 \mathrm{keV})$ is a collimated instrument with a $1^{\circ}$ field of view, and a background limited sensitivity of $\geqslant 10^{-12} \mathrm{erg} \mathrm{cm}^{-2} \mathrm{~s}^{-1}$ in the $2-10 \mathrm{keV}$ band, for a $100 \mathrm{ks}$ exposure. The $L O F T$ pointing visibility will assure a sky accessibility for these targets of $\sim 75 \%$, (LOFT Yellow Book). The requirements of our strategy define a $F_{\text {lim }} \sim 10^{-11} \mathrm{erg} \mathrm{cm}^{-2} \mathrm{~s}^{-1}$ in the $2-10 \mathrm{keV}$ band, which was then translated in the corresponding unabsorbed value in the $1-10 \mathrm{keV}$ band (the energy range adopted in our modeling). Again, we assume a one-day repointing delay. Figure 7 shows the expected rate of jetted TDEs for a LOFTlike mission as a function of redshift (right panel) and their 

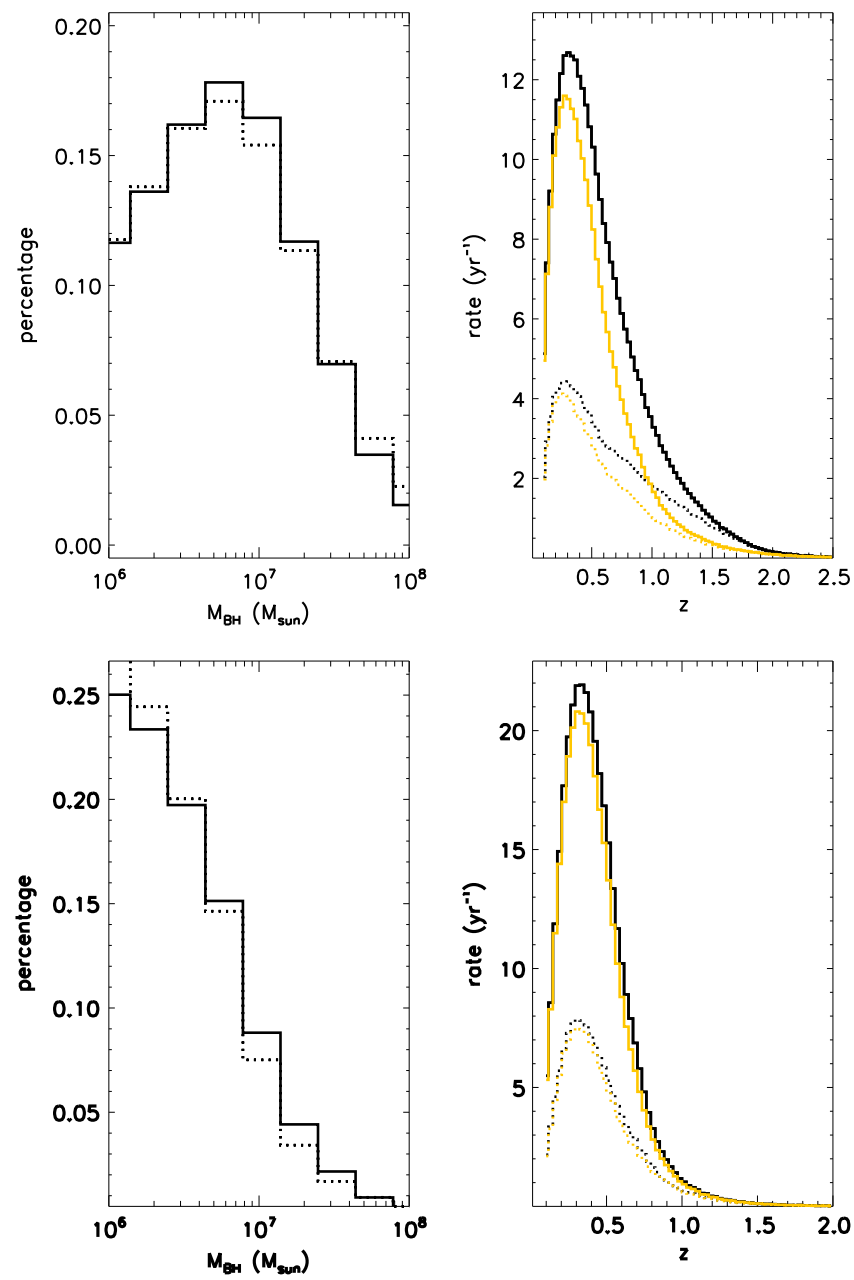

Figure 7. As in Figure 4, but for radio-triggered events repointed by a $L O F T$ mission.

mass distribution (left panel). The rate distributions are calculated under the BM model (top panels) and MDL model (bottom panels) for the radio modeling. In both cases, we found that the redshift distribution extends above $z=1\left(z_{\max } \approx\right.$ 1.2-1.7) (see Table 1), with most of the TDEs expected around $z \simeq 0.4$ (right panels). The peak rates are roughly between $\approx 4$ and 20 events per year. In the MDL model, because of the mass dependence of both the radio and X-ray luminosities, $\sim 25 \%$ of all events have $\mathrm{BHs}$ with masses $\approx 10^{6} M_{\odot}$, and events with $\mathrm{BH}$ masses $<10^{7} M_{\odot}$ dominate the redshift distribution at all epochs. In the BM model, instead, the TDE rate peaks at $\approx 10^{7} M_{\odot}$ (see the left panel in Figure 7$)$, with lighter BHs dominating at $z \leqslant z_{\text {peak }}$ (yellow lines in Figure 7, right panel). The behavior at higher $z$ fully reflects the one observed in the $\mathrm{BM}$ radio rates (see Figure 4 ). In total, a $L O F T$-like mission should be able to detect a sub-sample of radio TDEs between $\approx 130$ and $\simeq 350 \mathrm{yr}^{-1}$. Instead, very few objects per year are predicted if $\Gamma=20$. For these events, the mission broad energy band $(2-50 \mathrm{keV})$ should enable us to put tighter constraints on the energy budget of the X-ray component than is possible with the Athena instrument.

The price to pay for detecting more X-ray TDEs with a follow-up strategy is illustrated in Figure 5 (upper panel) where we compare the trigger distribution for the Einstein Probe (black lines) and the LOFT radio triggered (blue lines) samples. Most LOFT events are observed after 10 days from the beginning of the emission. ${ }^{9}$ In particular, high-redshift $z \approx 1$ events are all a couple of months old. Direct discovery of TDEs in X-rays is thus important for catching the event in its very early dynamical stages, when the jet has just formed and the disk may still be in the (largely unconstrained) superEddington regime.

\section{DISCUSSION}

The Swift/BAT discovery of Sw J1644 opened a window to a new class of X-ray and radio transients, which are optimal targets for future radio and X-ray surveys/instruments. The study of these objects allows us to investigate the formation of transient jets in extra-galactic sources. Moreover, there is the potential to discover quiescent SMBHs in distant galaxies and constrain the SMBH mass function. In this section, we qualitatively discuss our results and what we may learn from them. Any quantitative parameter investigation (for instance with a Fisher Matrix technique) is beyond the scope of this present paper, and will be presented in a follow-up work.

\subsection{Jet Efficiencies and Bulk Lorentz Factor}

Thus far, only two jetted TDEs have been detected, while the thermal candidates, related to the presence of an accretion disk, have been more numerous. The question then arises whether this is due to observational biases, highly collimated jets or to an intrinsic low efficiency of transient accretion disks to produce (luminous) jets.

To try and address this question, we could compare our predictions to the Swift/BAT observed rate $\left(\approx 0.3 \mathrm{yr}^{-1}\right)$ : our lower limit $\left(\approx 9 \mathrm{yr}^{-1}\right)$ is a factor of 30 higher. It is temptingand indeed it has been done in the literature-to reconcile this discrepancy by invoking a jet production efficiency of a few percent, since our calculations assume that each TDE is accompanied by a jet. ${ }^{10}$

However, there are several reasons why this inference should not be drawn. First, as discussed in Section 5.1.1, it is absolutely non-trivial to describe the characteristics (e.g., flux limit and sky coverage) of an effective Swift/BAT survey. We believe that our assumptions for the trigger, together with a $100 \%$ identification efficiency, give rates that are indicative of an upper limit. Second, BAT rate predictions, unlike those of other X-ray instruments consider here, strongly depend on the modeling of the early stage variability of the X-ray light curve (see Section 2). The onboard threshold we use is very close to the flux of the upper envelope of the light curve. We are therefore implicitly assuming that we can always trigger an event, by catching it at its maximum. However, since the flux varies by two orders of magnitude, our choice implies again an upper limit estimate of BAT rates. Finally, even if we trust our modeling of the BAT trigger and initial X-ray variability, uncertainties in the value of $\Gamma$ can account for the discrepancy. Thus far, we have considered a bulk Lorentz factor of two, since the radio measurements strongly support such a low $(\Gamma \sim 2-5)$ value. However, hard X-ray observations are

\footnotetext{
9 See discussion in Section 5.2.2.

${ }^{10}$ In our simplified description here, there are only two kinds of possible events: Sw J1644 with its own jet luminosity (i.e., a given jet energy efficiency $\left.\epsilon_{\mathrm{j}}\right)$ and events with no jet (i.e., $\epsilon_{\mathrm{j}}$ very small). In reality, there must be an intrinsic distribution of $\epsilon_{\mathrm{j}}$, with a tail of low-energy events that cannot be detected or failed to be launched at relativistic speeds.
} 
consistent with larger Lorentz factors $(\Gamma \leqslant 20$; Burrows et al. 2011), which will bring down our rates to the observed value (see Table 1). The consequence would be that the simultaneous hard X-ray and the radio emissions need to come from different regions - as already claimed (e.g., Zauderer et al. 2011). The picture may be that while the radio emission is produced from further out, after the jet has substantially decelerated, X-rays probes regions much closer to the central engine (Bloom et al. 2011). If that was true, X-ray detections and follow-ups would be further suppressed with respect to the expected SKA performance.

Unlike the previous comparison with BAT results, our predictions of the radio rates are consistent with the upper limits derived using with the NVSS + FIRST catalog (Bower 2011), for any $\Gamma \geqslant 2$. As a consequence, this comparison cannot provide us with further constraints on either $\Gamma$ or the jet efficiency. In the next future, surveys such as VLA Stripe 82, ASKAP, and VLASS will give tighter constraints on jetted TDEs thanks to the improved sensitivity (50 $\mu \mathrm{Jy}$ rms, Hodge et al. 2013) of the former and the wide field of view of the latter two surveys. In this case, our radio modeling predicts a few objects $\mathrm{yr}^{-1}$ (a few tens $\left.\mathrm{yr}^{-1}\right)$ to be detected by assuming $\Gamma=2$. Comparing predictions with (positive) observations will thus constrain possible combinations of $\Gamma$ and jet production efficiency.

As already discussed, the optical transient surveys PanSTARRS and LSST are expected to make significant advances in the study of TDEs. LSST will be a real breakthrough in this respect, surveying $2 \times 10^{4}$ square degrees of the southern sky. Thousands of objects are expected to be discovered at $z<1$ by catching their thermal light from the accretion disk or from the non-relativistic wind in the super-Eddington phase, surveying the same fraction of the sky every three days (Strubbe \& Quataert 2009; van Velzen et al. 2011a). However, optical extinction in galactic nuclei still introduces an observational bias in TDE discovery, although less significant with respect to that occurring in the UV band. As suggested by Strubbe \& Quataert (2009), infrared surveys will provide a complementary approach as it is the lower frequency energy range less affected by any source of obscuration.

Contrary to radio and X-ray emissions, the optical and infrared light are not expected to be relativistically beamed nor to be connected with jet emission. These features imply that a comparison between optical, X-ray, and radio-selected samples can help constrain both the TDE efficiency to produce jets and the relativistic Lorentz factor. This latter factor, when an X-ray sample is available, will help us assess the jet energy efficiency $\epsilon_{\mathrm{j}}$.

\subsection{Supermassive BH Masses}

To understand supermassive $\mathrm{BH}$ cosmic growth and its connection to the host galaxy, it is necessary to have a good understanding of which mass can be found in which galaxy and, more broadly, of the SMBH mass function as a function of redshift.

The detection and light modeling of a TDE event is a unique way to constrain the mass of an otherwise quiescent $\mathrm{BH}$, which is too distant to be detected by stellar dynamics. An attractive feature is that TDEs may occur in any type of galaxy, allowing for the detection of a broader range of SMBH hosts. For the light-curve modeling, a multi-wavelength approach can yield tighter constraints on the mass, since other parameters such as the jet energy, Lorentz factor and the stellar mass need to be simultaneously determined.

A perhaps more direct measurement of the $\mathrm{BH}$ mass can come from very fast X-ray variability, as the QPO observed in Sw J1644 (Reis et al. 2012). The prospect for detection of QPOs in such events is quite favorable for both an Athena- and a LOFT-like mission. If QPOs in TDEs were associated with the Keplerian frequency at the innermost stable orbit (as discussed in Reis et al. 2012), the highest rest-frame frequency should be of the order of $200 \mathrm{~s}$ for a BH mass of $10^{6} M_{\odot}$. This QPO frequency is easily within reach of both LOFT-like and Athena instruments (Feretti et al. 2014; Nandra et al. 2013). Longer oscillations are expected for more massive BHs $\left(\propto \sqrt{10^{6} / M_{\mathrm{BH}}}\right)$, whose detectability could be more complicated due to satellite orbit constraints (e.g., Earth occultation, South Atlantic Anomaly). However, provided that the QPO is persistent over a long period and the source is bright enough to remain above threshold for several cycles, a direct measure of such a QPO is also possible.

Another method to constrain the mass function may be to compare our rate distributions with future SKA triggered observations. As shown in Figure 3 (upper panel), there are still uncertainties in the $\mathrm{BH}$ mass function, which in turn affect our rate predictions (see Figure 3 (lower panel) and Figures 4 and 7).

\section{CONCLUSIONS}

We have investigated the best strategies to increase the sample of the new class of TDEs that was recently discovered by BAT. These events emitted non-thermal emission in X-ray and radio bands, probing a relativistic jet. Given the lack of statistics and of a solid theoretical framework for their nonthermal emission, we adopted a rather phenomenological approach to model their light curve. We fit the behavior of the best studied candidate, $\mathrm{Sw} \mathrm{J} 1644$, in both radio $(1.4 \mathrm{GHz})$ and X-rays $(1-10 \mathrm{keV})$, and we used the classical theory of TDEs to rescale the emission for different black hole and star masses. In the radio band, we also considered, alternatively, the blast wave model, usually adopted for GRBs. We then used a Monte Carlo code to compute their expected rate as a function of redshift and black hole mass. We considered both current and future radio and X-ray surveys/instruments. Since the characteristic temporal decay of a TDE event can be observed in X-ray, an identification is claimed only when the X-ray emission can be sampled in at least four light-curve bins with high signal-to-noise ratio, $\mathrm{S} / \mathrm{N} \geqslant 5$. When the TDE is detected in radio, we investigated a follow-up strategy for identification that required X-ray detectors to sample the light curve with almost the same requirements as above (but with a $\mathrm{S} / \mathrm{N} \geqslant 10$ ). To concretely explore future possibilities, we investigated in particular the expected performance of eRosita, Einstein Probe, Athena, a LOFT-like mission, and SKA operating in survey mode (SKA1-SUR).

Our major findings can be summarized as follows:

1. Results from current instruments (such as BAT and NVSS + FIRST catalogs) do not provide constraints on jet parameters or the jet production efficiency.

2. However, to reconcile BAT predictions with observations a $\Gamma \approx 20$ may be adopted, consistently with hard X-ray observations (Burrows et al. 2011). If this were true, $\mathrm{X}$ ray and radio emissions should come from two different 
regions, as already suggested on different bases (Zauderer et al. 2011). The predicted X-ray rates would also be suppressed by $(2 / 20)^{2}$ with respect to those in the radio band.

3. In the near future, VLA Stripe 82 survey, VLASS, and ASKAP-VAST may provide from a few to ten events per year, putting some constraints on possible combinations of bulk Lorentz $\Gamma$ and jet production efficiency.

4. Hundreds $(\Gamma=2)$ of Sw J1644-like objects per year are expected to be within reach of SKA1-SUR at $1.4 \mathrm{GHz}$. They can probe the distant Universe up to $z \sim 2.5$. These results differ from previous, more optimistic, predictions of thousands per year (for $\Gamma=2$ van Velzen et al. 2011)

5. Future $X$-ray surveys will provide a more modest sample, between several (eRosita) to a maximum of $\approx 240$ (EP) jetted events per year. With a highly collimated jet, with $\Gamma=20$, these numbers drop to a maximum of a few.

6. X-ray detections can be substantially enhanced, if a prompt follow-up of SKA candidate is adopted with an instrument with flux limit $\lesssim 10^{-11} \mathrm{erg} \mathrm{cm}^{-2} \mathrm{~s}^{-1}$ in the $1-10 \mathrm{keV}$ band over a four-day timescale. With that flux limit each SKA triggered event can have in principle an X-ray counterpart (see Figure 6). A suppression factor should be adopted if the X-ray emitting region would be moving with a larger Lorentz factor.

7. The sample of SKA preselected X-ray events can extend up to redshift $\sim 2$ for a X-ray instrument such as Athena and the LAD on board a LOFT-like mission. Instead, eRosita, the WFM on LOFT, and Einstein Probe samples will probe a redshift range only up to $z \lesssim 1$.

8. Despite the several advantages of a radio trigger, direct X-ray detections are the only way to study the early stages (<10 days) of the flare (see Figure 5).

Once TDE samples in different bands have been built up, the synergy between radio, X-rays, and optical can in principle constrain important physical quantities such as the jet luminosity, bulk Lorentz factor, the jet production efficiency, and the black hole mass function. These findings will inform theories of jet and disk formation from sudden accretion events and, on the other hand, of SMBH cosmological evolution.

We would like to thank Francesco Shankar for providing us with the black hole mass functions, and I. Prandoni and $\mathrm{H}$. Krimm for the interesting discussions about SKA and BAT observing strategies, respectively. Finally, we thank S. Van Velzen for the careful reading of this paper and his helpful comments. We thank the anonymous referee for helpful suggestions. This work made use of data supplied by the UK Swift Science Data Centre at the University of Leicester. I.D. is grateful for support by ASI (under contract I/021/12/0-186/12), INAF (under contract PRIN-INAF-2011 "Strong Gravity").

\section{REFERENCES}

Arcavi, I., Gal-Yam, A., Sullivan, M., et al. 2014, ApJ, 793, 38

Berger, E., Zauderer, A., Pooley, G. G., Soderberg, A. M., Sari, R., Brunthaler, A., \& Bietenholz, M. F. 2012, ApJ, 748, 36

Bloom, J. S., Giannios, D., Metzger, B. D., et al. 2011, Sci, 333, 203

Bower, G. C. 2011, ApJ, 732, L12

Burrows, D. N., Kennea, J. A., Ghisellini, G., et al. 2011, Natur, 476, 421
Campana, S. 2011, Natur, 480, 69

Cannizzo, J. K., Troja, E., \& Lodato, G. 2011, ApJ, 742, 32

Carilli, C. L., \& Rawlings, S. 2004, NewAR, 48, 979

Cenko, S. B., Krimm, H. A., Horesh, A., et al. 2012, ApJ, 753, 77

Chornock, R., Berger, E., Gezari, S., et al. 2014, ApJ, 780, 44

Cummings, J. R., Barthelmy, S. D., Beardmore, A. P., et al. 2011, GCN, 11823

Dewdney, P. E., Turner, W., Millenaar, R., et al. 2013, SKA1 System Baseline Design. Tech. Rep., SKA Program Development, Document Number: SKATEL-SKO-DD-001

Donato, D., Cenko, S. B., Covino, S., et al. 2014, ApJ, 781, 59

Donley, J. L., Brandt, W. N., Eracleous, M., \& Boller, T. 2002, ApJ, 124, 1308

Donnarumma, I., Rossi, E. M., Fender, R., et al. 2014, in The Transient Universe with the Square Kilometre Array Proc. Advancing Astrophysics with the Square Kilometre Array, PoS(AASKA14)054

Feretti, L., \& Prandoni, I. 2014, in Italian SKA White Book, ed. L. Feretti, I. Prandoni, et al. (INAF Press) ISBN 978-88-98985-00-5

Feroci, M., Stella, L., van der Klis, M., et al. 2012, ExA, 34, 415

Frail, D., Kulkarni, S. R., Ofek, E. O., Bower, G. C., \& Nakar, E. 2012, ApJ, 747,70

Frank, J., \& Rees, M. J. 1976, MNRAS, 176, 633

Gezari, S., Heckman, T., Cenko, S. B., et al. 2009, ApJ, 698, 1367

Gezari, S., Chornock, R., Rest, A., et al. 2012, Natur, 485, 217

Granot, J., \& Sari, R. 2002, ApJ, 568, 820

Guillochon, J., \& Ramirez-Ruiz, E. 2013, ApJ, 767, 25

Hayasaki, K., Stone, N., \& Loeb, A. 2013, MNRAS, 434, 909

Hallinan, G., Mooley, K. P., Kulkarniet, S. R., et al. 2013, Transient Science with the VLASS, VLASS White Paper

Hills, J. G. 1975, Natur, 254, 295

Holoien, T. W.-S., Prieto, J. L., Bersier, D., et al. 2014, MNRAS, 445, 3263

Hodge, J. A., Becker, R. H., White, R. L., \& Richards, G. T. 2013, ApJ, 769,125

Jose, J., Guo, Z., Long, F., et al. 2014, ATel, 6777

Kesden, M. 2012, PhRvD, 85, 4037

Khabibullin, I., Sazonov, S., \& Sunyaev, R. 2014, MNRAS, 437, 327

Kroupa, P. 2001, MNRAS, 322, 231

Komossa, S. 2002, RvMA, 15, 27

Krimm, H. A., Kennea, J. A., Holland, S. T., et al. 2011, ATel, 3384

Krimm, H. A., Holland, S. T., Corbet, R. H. D., et al. 2013, ApJS, 209, 14

Levan, A. J., Tanvir, N. R., \& Cenko, S. B. 2011, Sci, 333, 199

Lien, A., Sakamoto, T., Gehrels, N., et al. 2014, ApJ, 783, 24

Lodato, G., King, A. R., \& Pringle, J. E. 2009, MNRAS, 392, 332

Lodato, G., \& Rossi, E. M. 2011, MNRAS, 410, 359

Maksym, W. P., Lin, D., \& Irwin, J. A. 2014a, ApJL, 792, 29

Maksym, W. P., Irwin, J., Ulmer, M. P., et al. 2014b, AAS, 223, 406

Merloni, A., Predehl, P., Becker, W., et al. 2012, arXiv:1209.3114M

Merritt, D. 2013, CQGra, 30, 244005

Metzger, B. D., Giannios, D., \& Mimica, P. 2012, MNRAS, 420, 3528

Miller, J. M., Cenko, B., Gezari, S., et al. 2014, ATel, 6800

Murphy, T., Chatterjee, S., Kaplan, D. L., et al. 2013, PASA, 30, 6

Nandra, P., Barret, D., Barcons, X., et al. 2013, arXiv:1306.2307N

Narayan, R., Igumenshchev, I. V., Abramowicz, M. A., et al. 2003, PASJ, 55, L69

Phinney, E. S. 1989, Natur, 340, 595

Rees, M. J. 1988, Natur, 333, 523

Reis, R. C., Miller, J. M., Reynolds, M. T., et al. 2012, Sci, 337, 949

Rossi, E. M., \& Begelman, M. C. 2009, MNRAS, 392, 1451

Sądowski, A., Narayan, R., McKinney, J., \& Tchekhovskoy, A. 2014, MNRAS, 439, 503

Sari, R., Kobayashi, S., \& Rossi, E. M. 2010, ApJ, 708, 605

Saxton, C. J., Soria, R., Wu, K., \& Kuin, N. P. M. 2012, MNRAS, 422, 1625

Shankar, F., Weinberg, D. H., \& Miralda-Escudé, J. 2013, MNRAS, 428, 421

Soltan, A. 1982, MNRAS, 200, 115

Stone, N., \& Loeb, A. 2012, PhRvL, 108, 1302

Stone, N., Sari, R., \& Loeb, A. 2013, MNRAS, 435, 1809

Strubbe, L. E., \& Quataert, E. 2009, MNRAS, 400, 2070

Tchekhovskoy, A., Metzger, B. D., Giannios, D., \& Kelley, L. Z. 2014, MNRAS, 437, 2744

van Velzen, S., Farrar, G. R., Gezari, S., et al. 2011, ApJ, 741, 73

van Velzen, S., Frail, D., Körding, E., \& Falcke, H. 2011, MNRAS, 417, 51

van Velzen, S., Frail, D., Körding, E., \& Falcke, H. 2013, A\&A, 552, A5

Zauderer, B. A., Berger, E., Margutti, R., et al. 2013, ApJ, 767, 152

Zauderer, B. A., Berger, E., Soderberg, A. M., et al. 2011, Natur, 476, 425 